\title{
Chirality-Helicity of Cumulenes: A Non-Scalar Charge Density Derived Perspective
}

\author{
Hui Xing ${ }^{1}$, Alireza Azizi ${ }^{2}$, Roya Momen ${ }^{3}$, Tianlv Xu${ }^{1}$, Steven Kirk ${ }^{1}$, and Samantha \\ Jenkins ${ }^{1}$ \\ ${ }^{1}$ Hunan Normal University \\ ${ }^{2}$ Henan Normal University \\ ${ }^{3}$ Central South University
}

November 9, 2021

\begin{abstract}
We investigate the presence of helical character and chirality using a vector-based charge density perspective instead of energetic or structural measures. The vector-based perspective of the chemical bonding, constructed using the most preferred direction of charge density accumulation, finds the presence of induced symmetry-breaking for $\alpha, \omega$-disubstituted [4]cumulenes as the end groups are torsioned. The stress tensor trajectories $\mathrm{T} \sigma(\mathrm{s})$ are used to provide the additional symmetry-breaking required to quantify the degree and nature of the chirality and helical character. We find an absence of chirality for [4] cumulene but a very significant degree of axiality as demonstrated by the purely axial form of the $\mathrm{T} \sigma(\mathrm{s})$ indicating a lack of helical character. The S-1,5-dimethyl-[4]cumulene contains a very low degree of chiral character but significant axiality(helicity) resulting in a weakly helical morphology of the corresponding $\mathrm{T} \sigma(\mathrm{s})$. The $(-) \mathrm{S}(-),(+) \mathrm{S}(-)$ and $(+) \mathrm{S}(+)$ conformations of S-1,5-diamino-[4]cumulene contain very significant degrees of both chirality and helical character resulting in helical morphology of the corresponding $\mathrm{T} \sigma(\mathrm{s})$. The chirality assignments are in agreement with the Cahn-Ingold-Prelog (CIP) classifications for the (-)S(-), (+)S(-) and $(+) \mathrm{S}(+)$ conformations of S-1,5-diamino-[4]cumulene. We discuss the consequences for the T $\sigma(\mathrm{s})$ in locating chiral character in these molecules in future experiment investigations.
\end{abstract}

\section{Chirality-Helicity of Cumulenes: A Non-Scalar Charge Density Derived Perspective}

\section{Hui Xing ${ }^{1}$, Alireza Azizi ${ }^{1}$, Roya Momen ${ }^{2}$, Tianlv $\mathrm{Xu}^{1}$, Steven R. Kirk ${ }^{{ }^{*}}$ and Samantha} Jenkins $^{1^{*}}$

${ }^{1}$ Key Laboratory of Chemical Biology and Traditional Chinese Medicine Research and Key Laboratory of Resource

National and Local Joint Engineering Laboratory for New Petro-chemical Materials and Fine Utilization of Resources, College of Chemistry and Chemical Engineering, Hunan Normal University,

Changsha, Hunan 410081, China

${ }^{2}$ Central South University, College of Chemistry and Chemical Engineering, Lushan South Road Changsha, Hunan, CN 410083

email: steven.kirk@cantab.net

email: samanthajsuman@gmail.com

We investigate the presence of helical character and chirality using a vector-based charge density perspective instead of energetic or structural measures. The vector-based perspective of the chemical bonding, con- 
structed using the most preferred direction of charge density accumulation, finds the presence of induced symmetry-breaking for $a, \omega$-disubstituted [4]cumulenes as the end groups are torsioned. The stress tensor trajectories $\mathrm{T}_{\sigma}(s)$ are used to provide the additional symmetry-breaking required to quantify the degree and nature of the chirality and helical character. We find an absence of chirality for [4]cumulene but a very significant degree of axiality as demonstrated by the purely axial form of the $\mathrm{T}_{\sigma}(s)$ indicating a lack of helical character. The S-1,5-dimethyl-[4]cumulene contains a very low degree of chiral character but significant axiality(helicity) resulting in a weakly helical morphology of the corresponding $\mathrm{T}_{\sigma}(s)$. The $(-) \mathrm{S}(-),(+) \mathrm{S}(-)$ and $(+) \mathrm{S}(+)$ conformations of S-1,5-diamino-[4]cumulene contain very significant degrees of both chirality and helical character resulting in helical morphology of the corresponding $\mathrm{T}_{\sigma}(s)$. The chirality assignments are in agreement with the Cahn-Ingold-Prelog (CIP) classifications for the (-)S(-), (+) $\mathrm{S}(-)$ and (+) $\mathrm{S}(+)$ conformations of S-1,5-diamino-[4]cumulene. We discuss the consequences for the $\mathrm{T}_{\sigma}(s)$ in locating chiral character in these molecules in future experiment investigations.

\section{Introduction}

Earlier $^{1}$, the interdependence of steric-electronic factors for spiroconjugated molecules ${ }^{2}$ was determined to be more complex than that discernable from molecular structures alone, thereby calling into question the use of the Cahn-Ingold-Prelog (CIP) priority rules for determining the chiral configuration $(\mathrm{R} / \mathrm{S})^{3,4}$. Cumulenes are a class of linearly conjugated $\pi$-systems terminated by tri-coordinate carbon atoms ${ }^{5}$. The cumulene molecules are labeled by $n$, which refers to the number of cumulated double bonds; where the total number of carbon atoms is specified by $n+1$. For $[n$ ]cumulenes with even $n$ to form closed-shell $\pi$-systems the terminal carbon atoms need to be mutually perpendicular. These molecules will be referred to as "even" $[n$ ]cumulenes, see Scheme 1 . The frontier $\pi$ molecular orbitals (MOs) in unsubstituted even $[n]$ cumulenes are all explicitly degenerate, a consequence of the ideal $\mathrm{D}_{2 d}$ symmetry of the molecule. The degeneracy of the orbitals is lifted by $a, \omega$-disubstitution, which reduces the symmetry of the molecule to $\mathrm{C}_{2}$ or lower, rendering the molecule chiral. An axial torsion toward the achiral planar transition state may also break the degeneracy for a mutual rotation of the end carbons ${ }^{6-11}$. These molecules have helical frontier MOs as a direct result of the perpendicular end-groups, as investigated by Hendon et al ., ${ }^{12}$ an effect they named "electrohelicity".

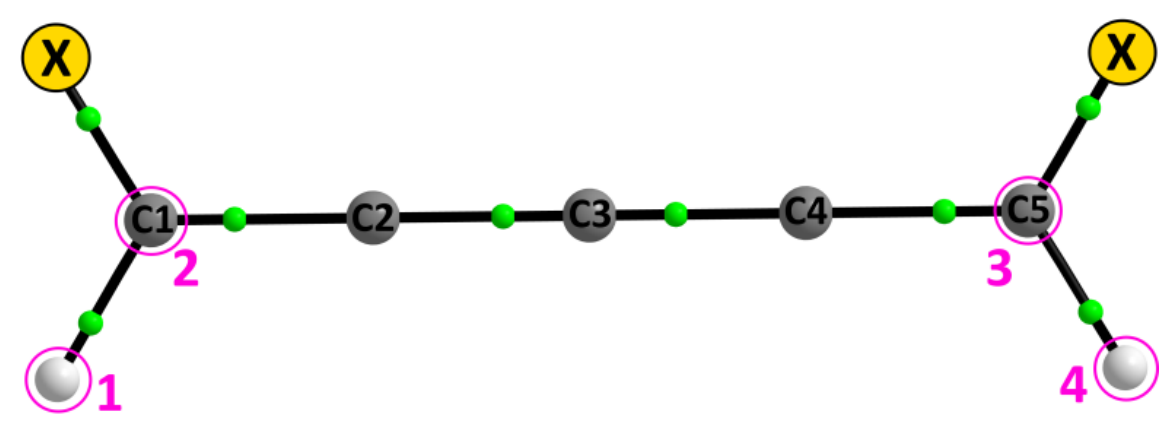

Scheme 1. The molecular graph of [4]cumulene, S-1,5-dimethyl-[4]cumulene and the (-)S(-), (+)S(-) and $(+) \mathrm{S}(+)$ conformations of S-1,5-diamino-[4]cumulene, where $\mathrm{X}=\left[\mathrm{H}, \mathrm{CH}_{3},(-) \mathrm{S}(-) \mathrm{NH}_{2},(+) \mathrm{S}(-) \mathrm{NH}_{2}\right.$, $(+) \mathrm{S}(+) \mathrm{NH}_{2}$ ]. The magenta circled atoms define the geometric dihedral angle specified in the order listed. The undecorated green spheres indicate the locations of the bond critical points ( $B C P \mathrm{~s})$.

Helical characteristics of stereoisomers were first proposed as the origin of chirality by Fresnel ${ }^{13}$ in 1851 , later this was demonstrated by optical experiments that show materials having different refractive indices for right (R) and left (S) circularly polarized light, known as circular dichroism ${ }^{14}$. These experiments were consistent with theories of optical activity that correlate the inherent helical identities with direction of rotation of the circularly polarized light ${ }^{15-17}$. A 'helix theory' was much later hypothesized by Wang for molecular chirality and chiral interaction who realized that evidence for this helical character was not provided by molecular geometries $^{18}$, or solely attributable to steric hindrance. Wang referred to the association between helical 
characteristics and chirality as the 'chirality-helicity equivalence'. Recent experiments by Beaulieu et al. on neutral molecules ${ }^{19}$, that utilize coherent helical motion of bound electrons, consistent with our previous work ${ }^{20}$ demonstrated the need for a better understanding of the behavior of the charge density redistribution. Consistent with Wang ${ }^{18}$, Banerjee-Ghosh et al. also recently demonstrated that charge density redistribution in chiral molecules, not spatial effects ${ }^{21}$, were responsible for an enantiospecific preference in electron spin orientation. Some of the current authors located, but did not quantify, for chiral compounds, the unknown chirality-helicity equivalence ${ }^{20}$ to enable the chiral discrimination of the $\mathrm{S}$ and $\mathrm{R}$ stereoisomers consistent with the naming schemes from optical experiments. The insufficiency of conventional (scalar) QTAIM ${ }^{22}$ (quantum theory of atoms in molecules) was also demonstrated for the chiral discrimination of $\mathrm{S}$ and $\mathrm{R}$ stereoisomers. If a helical response of the electronic charge density distribution is present on applying a bond torsion, then both non-axial (perpendicular to the bond-path) and axial (parallel to the bond-path) displacements of the torsional $\mathrm{C} 1-\mathrm{C} 2$ bond critical point $(B C P)$ will be found. Recently, the unknown chirality-helicity function, previously only located, was quantified consistent with photoexcitation circular dichroism experiments. We then used the chirality-helicity function to differentiate between steric effects due to eclipsed conformations and chiral behaviors in formally achiral species ${ }^{23}$.

In this investigation we will use NG-QTAIM (next generation QTAIM) to investigate each of the C-C $B C P$ $\mathrm{s}$ in terms of the 'orbital-like' $\{\mathrm{q}, \mathrm{q}$ ' $\}$ path-packets and the quantification of their wrapping around the bond-paths, the wrapping of these bond and the chirality and helicity properties using the stress tensor trajectory $\mathrm{T}_{\sigma}(s)$.

We use Bader's formulation of the stress tensor ${ }^{24}$ and NG-QTAIM due to the superior performance of the stress tensor compared with vector-based QTAIM in terms of clearer chiral discrimination of the S and $\mathrm{R}$ stereoisomers of lactic acid ${ }^{25}$. The NG-QTAIM chirality-helicity function will be applied to a range of [4]cumulenes to determine the presence of chirality and or helicity within the NG-QTAIM formalism.

\section{Theory and Methods}

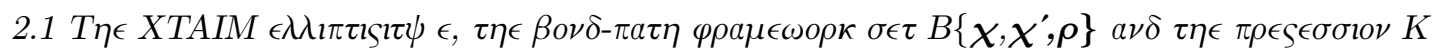

QTAIM is used to obtain critical points in the total electronic charge density distribution $\rho(\mathbf{r})$ by analyzing the gradient vector field [?] $\rho(\mathbf{r})^{22}$ where the complete set critical points are ordered according to the set of ordered eigenvalues $\lambda_{1}<\lambda_{2}<\lambda_{3}$, with corresponding eigenvectorse $e_{1}, e_{\mathbf{2}}, e_{\mathbf{3}}$ of the Hessian matrix of the total charge density $\rho(\mathbf{r})$. The complete set of critical points along with the bond-paths of a molecule or cluster is referred to as the molecular graph ${ }^{26}$. The most and least preferred directions of electron accumulation are $e_{\mathbf{2}}$ and $e_{1}$, respectively ${ }^{27-29}$; the eigenvector $e_{3}$ define the direction of the bond-path at the bond critical point $(B C P)$. The bond-path length (BPL) is defined as the length of the path traced out by the $e_{\mathbf{3}}$ eigenvector of the Hessian of the total charge density $\rho$ (r), passing through the $B C P$, along which $\rho(\mathbf{r})$ is locally maximal with respect to any neighboring paths ${ }^{30}$. The ellipticity $\varepsilon=\left|\lambda_{1}\right| /\left|\lambda_{2}\right|-1$, where $\lambda_{1}$ and $\lambda_{2}$ are negative eigenvalues of the corresponding $e_{1}$ and $e_{2}$ eigenvectors, respectively. The ellipticity $\varepsilon$ quantifies the relative accumulation of $\rho\left(\mathbf{r}_{\mathbf{b}}\right)$ in the $e_{\mathbf{2}}$ and $e_{\mathbf{1}}$ directions perpendicular to the bond-path at a $B C P$. The deviation from linearity, i.e. the shortest line separation, of a bond-path separating two bonded nuclei is defined as the dimensionless ratio (BPL - GBL)/GBL of the difference between the BPL and the geometric bond length (GBL) and the GBL.

The bond-path framework set $\mathrm{B}\{\mathrm{p}, \mathrm{q}, \mathrm{r}\}$ is comprised of three 'linkages'; $\mathrm{p}, \mathrm{q}$ and $\mathrm{r}$ associated with the $e_{\mathbf{1}}$, $e_{\mathbf{2}}$ and $e_{\mathbf{3}}$ eigenvectors, respectively is the NG-QTAIM interpretation of the chemical bond. The $\mathrm{p}$ and $\mathrm{q}$ are the 3-D paths that are constructed from the values of the least $\left(e_{\mathbf{1}}\right)$ and most $\left(e_{\mathbf{2}}\right)$ preferred directions of electronic charge density accumulation along the bond-path, referred to as $\mathrm{r}$. For further discussions on the construction of bond-path framework set B $\{\mathrm{p}, \mathrm{q}, \mathrm{r}\}^{31-41}$, see the Supplementary Materials S2 .

Two paths ( $\boldsymbol{q}$ and $\left.\mathrm{q}^{\prime}\right)$ are defined as being associated with the most preferred direction $e_{\mathbf{2}}$ eigenvector since $e_{\mathbf{2}}$ [?] $-e_{2}$ lies in the same plane for the same point on the bond-path $(\mathrm{r})$; correspondingly there are two paths associated with the $e_{\mathbf{1}}$ (p and p '), see equation (1 ). The pair of q - and q '-paths form packetshapes, that resemble orbitals, along the bond-path are referred to as a $\left\{q, q^{\prime}\right\}$ path-packet. The orientation, size and 
location of the $\left\{\mathrm{q}, \mathrm{q}^{\prime}\right\}$ path-packets relative to the associated $B C P$ indicate how much, which direction and whether a bond-path is twisted or linear. By definition, the $\left\{q, q^{\prime}\right\}$ path-packets are always orthogonal to the $\left\{\mathrm{p}, p^{\prime}\right\}$ path-packets. The form of the constituent $\left\{\mathrm{q}, \mathrm{q}^{\prime}\right\}$ path-packets can be used to provide a 3-D interpretation of bonding as a mixture of the following concepts: double bond, single bond, covalent, ionic or diradical. Note that, double and single bonds for shared-shell $\mathrm{C}-\mathrm{C} B C P$ s correspond to values of the C-C $B C P$ ellipticity $\varepsilon>0.25$ and $\varepsilon<0.25$ respectively, also the $\left\{q, q^{\prime}\right\}$ path-packets have no enclosed area around the $B C P$ for values of ellipticity $\varepsilon=0$.

Using $n$ points $r_{\mathbf{i}}$ along the bond path $\mathrm{r}$ (associated with eigenvector $e_{\mathbf{3}}$ ) and defining $\varepsilon_{\mathrm{i}}$ as the ellipticity at this point, one can draw vectors $\mathrm{q}_{\mathrm{i}}$ and $\mathrm{p}_{\mathrm{i}}$, scaled by $\varepsilon_{\mathrm{i}}$, originating at this point. The tips of these vectors $\left(q_{i}\right.$ and $p_{i}$ ) define the paths $p$ and $q$, where the form of $p_{i}$ and $q_{i}$ is defined as follows:

$\mathrm{p}_{\mathrm{i}}=\mathrm{r}_{i}+\varepsilon_{i} \boldsymbol{e}_{\mathbf{1}, i}, \mathrm{q}_{\mathrm{i}}=\mathrm{r}_{i}+\varepsilon_{i} \boldsymbol{e}_{\mathbf{2}, i}(\mathbf{1})$

We will now define the extent to which the $\left\{\mathrm{p}, p^{\prime}\right\}$ path-packet constructed from the $e_{\mathbf{1}}$ eigenvector wraps i.e.precesses about a C-C bond-path, see the left panel of Scheme $\mathbf{1}$. For the $\left\{\mathrm{p}, p^{\prime}\right\}$ path-packet, defined by the $e_{\mathbf{1}}$ eigenvector, we wish to follow the extent to which the $\left\{\mathrm{p}, p^{\prime}\right\}$ path-packet precesses about a C-C bond-path by defining the precession $\mathrm{K}$ for bond-path-rigidity ${ }^{42-44}$ :

$\mathrm{K}=1-\cos ^{2} \alpha$, where $\cos \alpha=e_{\mathbf{1}}[$ ? $] \boldsymbol{u}$ and 0 [?] $\mathrm{K}$ [?] 1 (2)

Where $u$ is the reference direction defined by the $e_{\mathbf{1}}$ eigenvector at the C1-C2 BCPfor each of the unsubstituted and substituted cumulenes for each choice of the geometrical dihedral angle $\left(0.0^{\mathrm{O}}, 15.0^{\mathrm{O}}, 75.0^{\mathrm{O}}\right.$ and $90.0^{\mathrm{o}}$ ). Considering the extremes of the precession $\mathrm{K}$, with $\alpha$ defined by equation $(\mathbf{2})$, for $\mathrm{K}=0$, there is maximum alignment of the $e_{1}$ eigenvector with the reference direction $u$, the least facile direction for the $\mathrm{C} 1-\mathrm{C} 2 B C P$. When $\mathrm{K}=1$ we have the maximum degree of alignment with the $e_{\mathbf{2}}$ eigenvector, the most facile direction. In other words, $\mathrm{K}=0$ and $\mathrm{K}=1$ indicate $\mathrm{C}-\mathrm{C}$ bond-paths with the lowest and highest tendencies towards $\mathrm{C}-\mathrm{C}$ bond-path-flexibility, respectively. The precession $\mathrm{K}$ is determined relative to the $B C P$, in either direction along the C-C bond-path towards the nuclei at either end of the bond-path using an arbitrarily small spacing of $e_{\mathbf{1}}$ eigenvectors. If we choose the precession $\mathrm{K}$ of the $\left\{\mathrm{p}, p^{\prime}\right\}$ path-packet about the $\mathrm{C}-\mathrm{C}$ bond-path when the $\pm e_{\mathbf{1}}$ eigenvector is parallel to $u$, the $B C P$ will have minimum facile character, i.e. bond-path-rigidity. By following the variation of the precession $\mathrm{K}$ we can quantify the degree of facile character of a $B C P$ along an entire C-C bond-path.

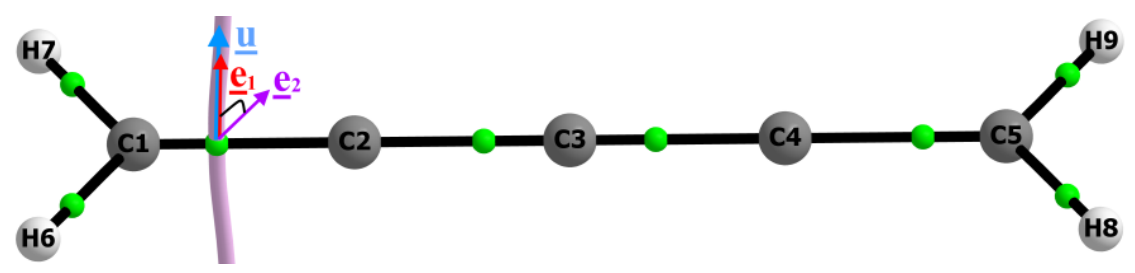

Scheme 2. The construction of the $\left\{p, \boldsymbol{p}^{\prime}\right\}$ precession $\mathrm{K}$ and $\left\{q, \boldsymbol{q}^{\prime}\right\}$ precession $\mathrm{K}^{\prime}$ corresponding to the unsubstituted cumulene molecular graph. The unit vector $u$ (blue arrow) is defined by the $e_{\mathbf{1}}$ eigenvector (red arrow) of the $\mathrm{C} 1-\mathrm{C} 2 B C P$, the $e_{\mathbf{2}}$ eigenvector (purple arrow) is orthogonal to $e_{\mathbf{1}}$. The pale magenta line indicates the interatomic surface paths (IAS) that originate at the $B C P$. The undecorated green spheres indicate the locations of the $B C P$ s.

For the precession of the $\left\{\mathrm{q}, q^{\prime}\right\}$ path-packet, defined by the $e_{\mathbf{2}}$ eigenvector, about the bond-path, $\beta=(\pi / 2$ $-\alpha)$ and $\alpha$ is defined by equation (3) see Scheme 1, we can write an expression K':

$\mathrm{K}^{\prime}=1-\cos ^{2} \beta$, where $\cos \beta=e_{\mathbf{2}}\left[\right.$ [?] $\boldsymbol{u}, \beta=(\pi / 2-\alpha)$ and 0 [?] $\mathrm{K}^{\prime}$ [?] 1 (3)

Note, for the general case the $e_{\mathbf{3}}$ eigenvector is defined along the bond-path and is not perpendicular to the reference direction $u$, see Scheme $\mathbf{1}$. For K' $=0$ we have a maximum degree of facile character and for $\mathrm{K}$ $=1$ we have the minimum degree of facile character. 
The presence of values of the precession $\mathrm{K}^{\prime}$ in the range 0 [?] $\mathrm{K}^{\prime}$ [?] 1 indicates polarization of the electron density $\rho(\mathbf{r})$ associated with the bond-path in terms of the changing orientation of the $e_{2}$ eigenvectors, from parallel to perpendicular, including intermediate orientations of the $e_{\mathbf{2}}$ eigenvector. Consequently, there will be a range of 'mixed' bond types within the limits of the rigid shared-shell character $\mathrm{K}$ ' $=1$, characteristic of sigma bonds and flexible closed-shell character $\mathrm{K}^{\prime}=0$, characteristic of hydrogen bonding.

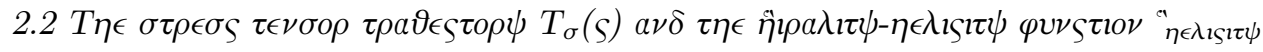

The procedure to generate the stress tensor trajectories $\mathrm{T}_{\sigma}(s)$ is provided in the Supplementary Materials S1. In this investigation we will use Bader's formulation of the stress tensor ${ }^{24}$ within the QTAIM partitioning which is a standard option in the QTAIM AIMAll ${ }^{45}$ suite. Earlier, we demonstrated that the most preferred direction for bond displacement, corresponding to most preferred direction of electronic charge density displacement, is the $\boldsymbol{\varepsilon}_{1 \sigma}$ eigenvector of the stress tensor ${ }^{31}$. Recently, we established the stress tensor trajectory $\mathrm{T}_{\sigma}(s)$ classifications for the $\mathrm{S}$ and $\mathrm{R}$ stereoisomers of lactic acid and alanine based on the counterclockwise $(\mathrm{CCW})$ vs. clockwise $(\mathrm{CW})$ torsions for the $e_{1 \sigma} . \mathrm{dr}$ components of $\mathrm{T}_{\sigma}(s)^{20}$. The calculation of the stress tensor trajectory $\mathrm{T}_{\sigma}(s)$ for the torsional $B C P$ is undertaken the frame of reference defined by the mutually perpendicular stress tensor eigenvectors $\left\{ \pm e_{\mathbf{1} \sigma}, \pm e_{\mathbf{2} \sigma}, \pm e_{3 \sigma}\right\}$ at the torsional $B C P$, corresponding to the geometric dihedral angle $=0.0^{\circ}$. This frame of reference is referred to as the stress tensor trajectory space (also named $\mathrm{U}_{\sigma}$-space). All the subsequent points along the $\mathrm{T}_{\sigma}(s)$ for dihedral torsion angles in the range $-180.0^{\mathrm{O}}[?] \vartheta[?]+180.0^{\mathrm{O}}$, use this frame of reference. We adopt the convention that $\mathrm{CW}$ circular rotations correspond to the range $-180.0^{\circ}[?] \vartheta[?] 0.0^{\circ}$ and CCW circular rotations to the range $0.0^{\circ}[?] \vartheta[?]+180.0^{\circ}$. Consistent with optical experiments, we defined from the stress tensor trajectory $\mathrm{T}_{\sigma}(s)$ that $\mathrm{S}$ (left-handed) character is dominant over $\mathrm{R}$ character (right-handed) for values of $(\mathrm{CCW})>(\mathrm{CW})$ components of the stress tensor trajectory $\mathrm{T}_{\sigma}(s)$. The stress tensor trajectory $\mathrm{T}_{\sigma}(s)$ are constructed using the change in position of the $B C P$, referred to as $\mathbf{d r}$, for all displacement steps $\mathbf{d r}$ of the calculation. Each finiteBCP shift vector $\mathbf{d r}$ is mapped to a point $\left\{\left(e_{\mathbf{1}} \sigma[?] \mathbf{d r}\right),\left(e_{\mathbf{2}} \sigma[?] \mathbf{d r}\right),\left(e_{\mathbf{3}} \sigma[?] \mathbf{d r}\right)\right\}$ in sequence, forming the stress tensor trajectory $\mathrm{T}_{\sigma}(s)$, constructed from the vector dot products (the dot product is a projection, or a measure of vectors being parallel to each other) of the stress tensor trajectory $\mathrm{T}_{\sigma}(s)$ evaluated at the $B C P$. The projections of $\mathbf{d r}$ in $\mathrm{U}_{\sigma}$-space are associated with the bond torsion: $e_{1 \sigma} \cdot \mathbf{d r}$-bond-twist, $e_{\mathbf{2} \sigma} \cdot \mathbf{d r}$ - bond-flexing and $e_{\mathbf{3} \sigma} \cdot \mathbf{d r}$-bond-axiality ${ }^{20,46-51}$. Note previously we referred to $e_{\mathbf{3} \sigma} \cdot \mathbf{d} \mathbf{r}$ as bond-axiality or bond-anharmonicity.

The bond-twist $\mathrm{T}_{\sigma}$ is defined by the difference in the maximum projections (the dot product of the stress tensor $\boldsymbol{\varepsilon}_{1 \sigma}$ eigenvector and the $B C P$ shiftdr $)$ of the stress tensor trajectory $\mathrm{T}_{\sigma}(s)$ values between the CCW and CW torsions $\mathrm{T}_{\sigma}=\left[\left(\boldsymbol{\varepsilon}_{1 \sigma}[;] \delta \rho\right)_{\mu \alpha \xi}\right]_{\mathrm{CCW}^{-}}\left[\left(\boldsymbol{\varepsilon}_{1 \sigma}[;] \delta \rho\right)_{\mu \alpha \xi}\right] \mathrm{CW}$. The bond-twist $\mathrm{T}_{\sigma}$ quantifies the bond torsion direction CCW vs. CW, i.e. circular displacement, where $\boldsymbol{\varepsilon}_{1 \sigma}$ corresponds to the most preferred direction of charge density accumulation. Note, previously we referred to bond-twist $\mathrm{T}_{\sigma}$ as the chirality $\mathrm{C}_{\sigma}$ because a single dominant torsion bond for the molecule was being used to determine the chirality $\mathrm{C}_{\sigma}$ properties of that molecule. In this investigation therefore, we refer to bond-twist $\mathrm{T}_{\sigma}$ instead of chirality $\mathrm{C}_{\sigma}$. Each of the C-C BCP s analyzed however, will still be assigned $\boldsymbol{\Sigma}_{\boldsymbol{\sigma}}$ or $\mathbf{P}_{\boldsymbol{\sigma}}$ character.

The least preferred displacement of a $B C P$ in the $\mathrm{U}_{\sigma}$-space distortion set $\left\{\mathrm{T}_{\sigma}, \mathrm{F}_{\sigma}, \mathrm{A}_{\sigma}\right\}$ is the bond-flexing $\mathrm{F}_{\sigma}$, defined as $\mathrm{F}_{\sigma}=\left[\left(\boldsymbol{\varepsilon}_{2 \sigma}[;] \delta \rho\right)_{\mu \alpha \xi}\right]^{\cdots "} \Omega^{-}\left[\left(\boldsymbol{\varepsilon}_{2 \sigma}[;] \delta \rho\right)_{\mu \alpha \xi}\right]^{\prime} \Omega$. The bond-flexing $\mathrm{F}_{\sigma}$ provides a measure of the 'flexing-strain' that a bond-path is under when, for instance, subjected to an external force such as an E -field.

Previously we used the term helicity $\mathrm{B}_{\sigma}$, defined as $\mathrm{B}_{\sigma}=\left[\left(\boldsymbol{\varepsilon}_{3 \sigma}[;] \delta \rho\right)_{\mu \alpha \xi}\right]_{\mathbf{C C W}}-\left[\left(\varepsilon_{3 \sigma}[;] \delta \rho\right)_{\mu \alpha \xi}\right.$ ]$_{\mathbf{C W}}$ quantifies the direction of axial displacement of the bond critical point $(B C P)$ in response to the bond torsion (CCW vs. CW), i.e. the sliding of the $B C P$ along the bond-path ${ }^{51}$. In this investigation to avoid confusion with work on the helical orbitals of $\left[n\right.$ ]cumulenes we will use the term axiality $\mathrm{A}_{\sigma}[$ ? $] \mathrm{B}_{\sigma}$. The sign of the chirality determines the dominance of $\boldsymbol{\Sigma}_{\boldsymbol{\sigma}}\left(\mathrm{T}_{\sigma}>0\right)$ and $\mathbf{P}_{\boldsymbol{\sigma}}\left(\mathrm{T}_{\sigma}<0\right)$ character, see Tables $\mathbf{2}$. The axiality $\mathrm{A}_{\sigma}$ determines the dominance of $\boldsymbol{\Sigma}_{\boldsymbol{\sigma}}$ or $\mathbf{P}_{\sigma}$ character with respect to the $B C P$ sliding along the bond-path as a consequence of the bond-torsion. Note the use of the subscript " $\sigma$ " used for the $\boldsymbol{\Sigma}_{\sigma}$ or $\mathbf{P}_{\sigma}$ assignments to denote calculation by the stress tensor trajectory $\mathrm{T}_{\sigma}(s)$. 
$\mathrm{A}_{\sigma}>0$ indicates dominant $\boldsymbol{\Sigma}_{\boldsymbol{\sigma}}$ character and the converse is true for $\mathrm{A}_{\sigma}<0$. The reason for calculating the $\mathrm{T}_{\sigma}(s)$ by varying the torsion $\vartheta$ is to detect values of the axiality $\mathrm{A}_{\sigma}[?] \quad 0$, i.e. $B C P$ sliding.

The chirality-helicity function $\mathrm{C}_{\text {helicity }}$ is formed from the simple arithmetic product of the bond-twist $\mathrm{T}_{\sigma}$ and the axiality $\mathrm{A}_{\sigma}$. The presence of a helical or chiral response, is determined by $\mathrm{A}_{\sigma}[?] 0$ or $\mathrm{T}_{\sigma}[?] 0$ of the torsional $B C P$ to the applied torsion $\vartheta$ coinciding with a helical stress tensor trajectory $\mathrm{T}_{\sigma}(s)$, for the conventionally chiral molecules such as lactic acid and alanine. In this investigation we will determine the $\mathrm{C}_{\text {helicity }}$ for all four of the C-C BCP s along each of the [4]cumulene variant molecular graphs.

\section{Computational Details}

The original molecular structures of the [4]cumulene, S-1,5-dimethyl-[4]cumulene and the were geometryoptimized at the PBE1PBE/aug-cc-pVTZ level of theory with empirical GD3-BJ ${ }^{52,53}$ dispersion correction using Gaussian09 rev E.01 ${ }^{54}$ with default (tight) geometrical convergence criteria, an 'ultrafine' DFT integration grid and an SCF convergence criterion of $10^{-10}$. All subsequent single-point calculations were also performed using these DFT parameters, basis set and convergence parameters. For the amine-substituted cumulene we start out from three of the six conformations ${ }^{55}$. At approximately $-90^{\circ}(-) \mathrm{S}(-)$ is rotated into $(-) \mathrm{R}(-)$ conformation. Likewise, at approximately $-90^{\circ}(+) \mathrm{S}(+)$ is rotated into $(+) \mathrm{R}(+)$ conformation; at approximately $-90^{\circ}(+) \mathrm{S}(-)$ is rotated into $(+) \mathrm{R}(-)$ conformation. Variants of the geometry-optimized [4]cumulenes were then generated by 'freezing' all bond lengths and angles, except for the H-C1-C2-C3-C4 -C5-H geometrical dihedral angle, where $\mathrm{C} 1$ and $\mathrm{C} 5$ are the carbon atoms at each end of the five-carbon atom chain which were constrained to take specific values. Geometrical dihedral angle $=0.0^{\circ}$ was chosen as the angle reference. Hence, only the end groups attached to the $\mathrm{C} 5$ carbon atom were allowed to 'rigidly' rotate about the dihedral axis - all other molecular geometrical degrees of freedom except the chosen dihedral angle were fixed. Positive and negative values of the dihedral angle were chosen in the range $-180.0^{\circ}$ [?] 0.0deg [?] +180.0 . For each dihedral angle, single-point calculations were performed and the resulting wavefunction analyzed using AIMAll ${ }^{45}$. The $\{\mathrm{q}, \mathrm{q}$ ' $\}$ path-packets and eigenvector precession profiles were generated from the eigenvectors of the Hessian of the total charge density using the 'framepath' and 'pathtool' codes from our in-house code suite QuantVec-Tools ${ }^{56}$. In all cases, the precession profile reference direction was chosen to be the $e_{\mathbf{1}}$ eigenvector of the Hessian of the charge density at the C1-C2 $B C P$. The $\left\{q_{,}, \mathrm{q}\right.$ ' $\}$ path-packets were visualized using the 'topviz' interactive GUI, also part of QuantVec-Tools, based on the Python 3 'mayavi2' visualization library ${ }^{57}$.

\section{Results and Discussion}

\subsection{The bond-path framework set $B\{\boldsymbol{q}, \boldsymbol{q}, \boldsymbol{r}\}$ and the precession $K$ of [4]cumulene and variants}

Examination of the distance measures reveals that none of the $\mathrm{C}-\mathrm{C} B C P$ s are located at the mid-points of the C-C BCPbond-paths as may be expected, see Table $\mathbf{1}$, further scalar measures, include distances and the relative energies [?] E are provided in the Supplementary Materials S2 . The locations of the C-C $B C P$ s are rather similar for the [4]cumulene and S-1,5-dimethyl-[4]cumulene molecular graphs, where the terminal C1-C2 $B C P$ and $\mathrm{C} 4-\mathrm{C} 5 B C P$ are located closest to the corresponding terminal $\mathrm{C} 1$ and $\mathrm{C} 5$ atom respectively, than to the $\mathrm{C} 2$ or $\mathrm{C} 4$ atoms. The central $\mathrm{C} 2-\mathrm{C} 3 B C P$ and $\mathrm{C} 3-\mathrm{C} 4 B C P$ display the opposite effect in that both these $B C P \mathrm{~s}$ are located closest to the more central $\mathrm{C} 3$ atom. The effect of the (-) $\mathrm{S}(-)$, $(+) \mathrm{S}(-)$ and $(+) \mathrm{S}(+)$ conformations of $\mathrm{S}-1,5$-diamino-[4]cumulene is to reverse the positions of the $B C P \mathrm{~S}$ where the terminal $B C P$ s are located closest to the $\mathrm{C} 2$ and $\mathrm{C} 4$ atoms for the $\mathrm{C} 1-\mathrm{C} 2 B C P$ and $\mathrm{C} 4-\mathrm{C} 5 B C P$ respectively. The positions of the central $\mathrm{C} 2-\mathrm{C} 3 \mathrm{BCP}$ and $\mathrm{C} 3-\mathrm{C} 4 \mathrm{BCP}$ are also located closest to the $\mathrm{C} 2$ and C4 atoms respectively.

Table 1. The partial bond-path lengths (in a.u.) of cumulene and the variants for values of the geometric dihedral angle $=0.0^{\circ}$.

(C1-BCP,BCP-C2) (C2-BCP,BCP-C3) (C3-BCP,BCP-C4) (C4-BCP,BCP-C5)[4]cumulene 0.9293,1.5379 $1.4434,0.96000 .9600,1.44341 .5379,0.9293 S$-1,5-dimethyl-[4]cumulene 0.9381,1.5355 1.4392,0.9657 0.9657, $1.43921 .5354,0.9381$ (-)S(-) S-1,5-diamino-[4]cumulene 1.3456, 1.1454 0.9942,1.4096 1.4096,0.9943 1.1449, 
1.3458(+)S(-) S-1,5-diamino-[4]cumulene 1.3514, 1.1395 0.9903,1.4134 1.4134,0.9905 1.1396, 1.3511(+)S(+) S-1,5-diamino-[4]cumulene 1.3569, 1.1340 0.9868,1.4170 1.4173,0.9866 1.1396, 1.3573

The bond-path framework set $\mathrm{B}\left\{\mathrm{q}, \mathrm{q}^{\prime}, \mathrm{r}\right\}$ comprise the most preferred $\left\{\mathrm{q}, \mathrm{q}^{\prime}\right\}$ path-packets of $[4]$ cumulene, S-1,5-dimethyl-[4]cumulene and the (-) S(-), (+)S(-) and (+) S(+) conformations of S-1,5-diamino-[4]cumulene at the geometrical dihedral angle of $0.0^{\circ}$ are presented in Figure 1. At a geometrical dihedral angle of $0.0^{\circ}$ the $\mathrm{C} 2-\mathrm{C} 3 B C P$ and $\mathrm{C} 3-\mathrm{C} 4 B C P$, on the basis of the size of the $\left\{\mathrm{q}, \mathrm{q}^{\prime}\right\}$ path-packets (BCPellipticity $\varepsilon=0.15$ ), are considered to be single bond-like and C1-C2BCP and C4-C5 $B C P$ are double bond-like (BCPellipticity $\varepsilon=0.42)$ for both the [4]cumulene, S-1,5-dimethyl-[4]cumulene molecular graphs, see Figure 1(a-b) . The corresponding $\left\{\mathrm{q}, \mathrm{q}^{\prime}\right\}$ path-packets of the $\mathrm{C} 2-\mathrm{C} 3 \mathrm{BCP}$ and $\mathrm{C} 3-\mathrm{C} 4 \mathrm{BCP}$ contain increasing degrees of polar character, indicated by the asymmetrical character of the $\left\{\mathrm{q}, \mathrm{q}^{\prime}\right\}$ path-packets, for the $(-) \mathrm{S}(-),(+) \mathrm{S}(-)$ and $(+) \mathrm{S}(+)$ conformations of S-1,5-diamino-[4]cumulene, see Figure 1(c-e) . A lower degree of asymmetrical character of the $\left\{\mathrm{q}, \mathrm{q}^{\prime}\right\}$ path-packets is apparent for geometric dihedral angles $=15.0^{\circ}, 75.0^{\circ}$ and $90.0^{\circ}$, see theSupplementary Materials S2 . The $\{\mathrm{p}, \mathrm{p}$ ' $\}$ path-packets that correspond to the least preferred directions of charge density accumulation are provided in the Supplementary Materials S3.

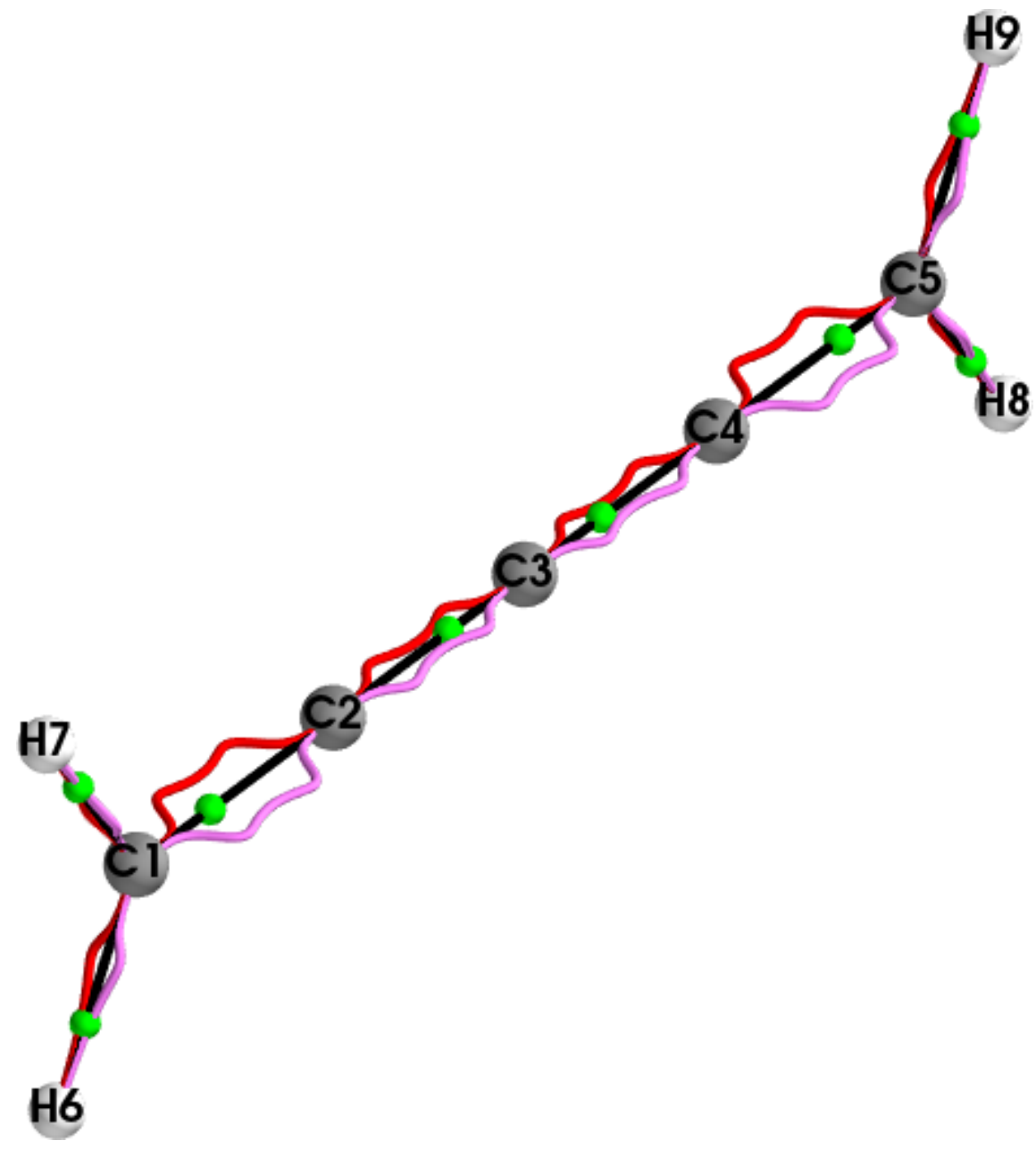




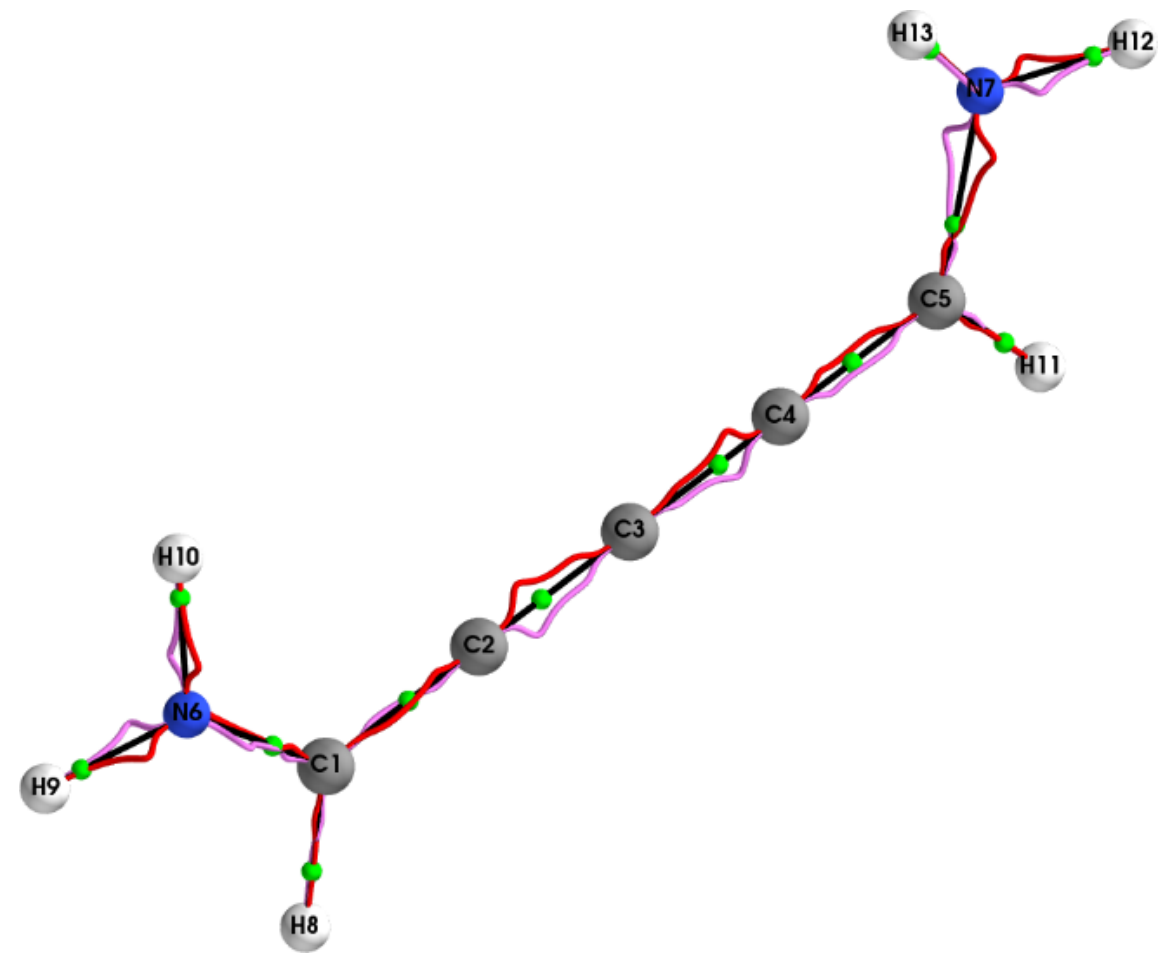

(a) (b)

(c) (d)

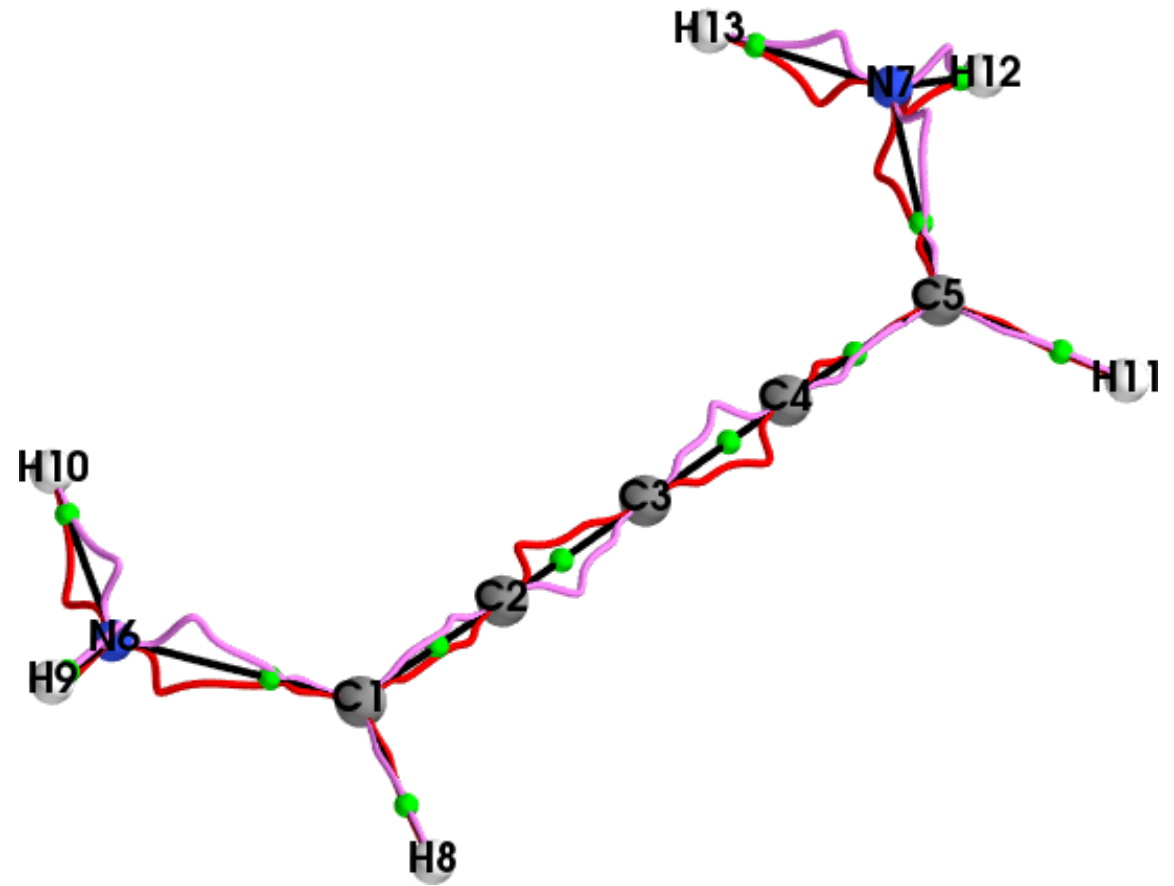

(e) 
Figure 1. The QTAIM $\left\{\boldsymbol{q}, \boldsymbol{q}^{\prime}\right\}$ path-packets of the [4]cumulene, S-1,5-dimethyl-[4]cumulene and the (-)S(-), $(+) \mathrm{S}(-)$ and $(+) \mathrm{S}(+)$ conformations of S-1,5-diamino-[4]cumulene molecular graphs are presented for the geometrical dihedral angle $=0.0^{\circ}$ in sub-figures (a-e) respectively.
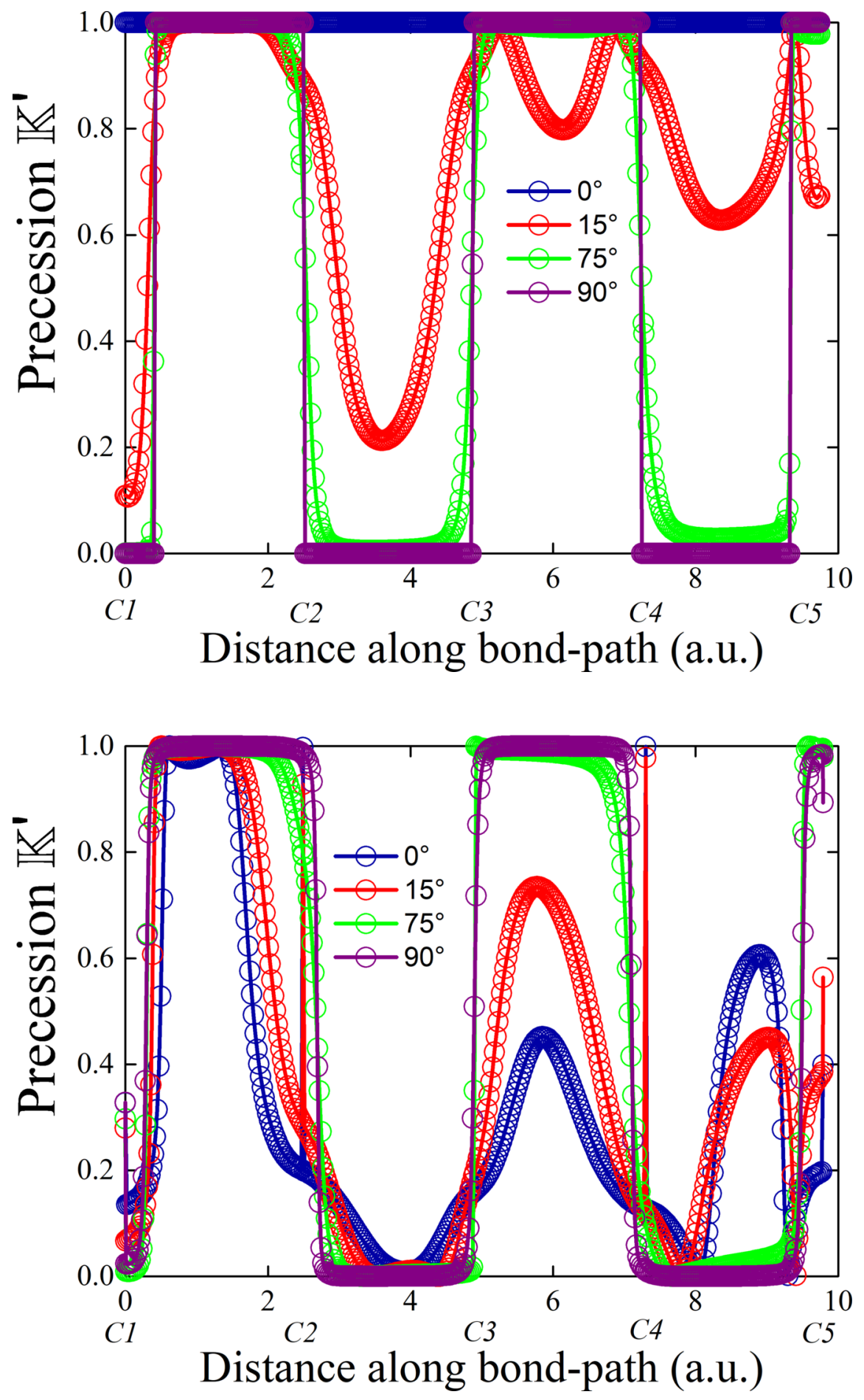

(a) (b) 
(c) $(\mathrm{d})$

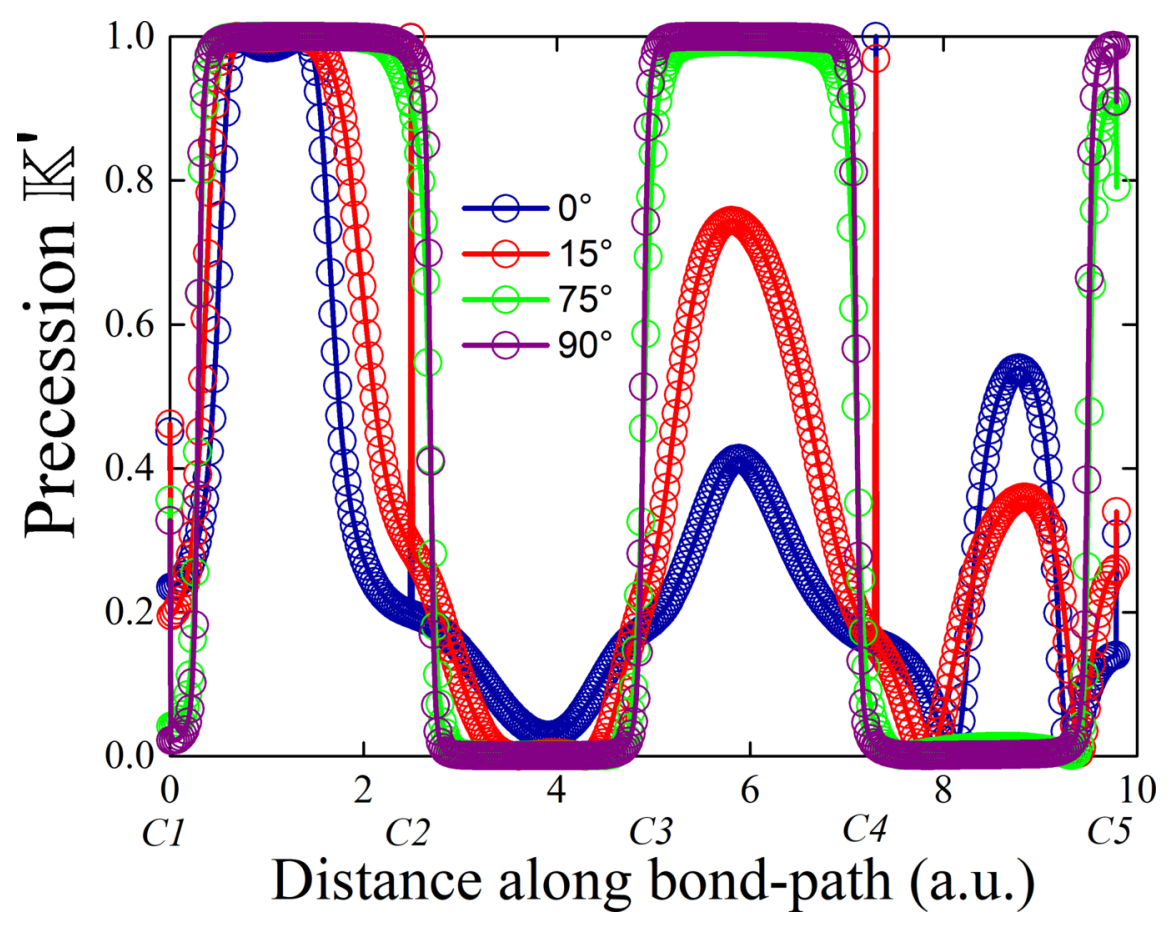

(e)

Figure 2. The variation of the C1-C2 $B C P$, C2-C3BCP , C3-C4 $B C P$ and C4-C5 $B C P$ bond-path precessions K' along bond-path for geometric dihedral angles $=0.0^{\mathrm{O}}, 15.0^{\mathrm{O}}, 75.0^{\mathrm{O}}$ and $90.0^{\mathrm{O}}$ are presented end-to-end $C$ 1- $C$ 2- $C$ 3- $C$ 4- $C 5$ for [4]cumulene, S-1,5-dimethyl-[4]cumulene and the (-)S(-), (+)S(-) and $(+) \mathrm{S}(+)$ conformations of S-1,5-diamino-[4]cumulene in sub-figures(a-e) respectively.

Note, the precession K' provides a directional interpretation of chemical bonding, see Figure $\mathbf{2}$. The corresponding $\mathrm{K}$ for the wrapping of the $\{\mathrm{p}, \mathrm{p}$ ' $\}$ path-packets around the C-C $B C P$ bond-paths $\mathrm{r}$ are provided in the Supplementary Materials S4 . The presence of values of K' $=1$ for the entire C1$\mathrm{C} 2-\mathrm{C} 3-\mathrm{C} 4-\mathrm{C} 5$ chain indicates the presence of bonding that possesses the deformation characteristics of rigid shared-shell $B C P$ bonding and a $\mathrm{Q}_{\text {rigidity }}=1.000$. Conversely, values of $\mathrm{K}^{\prime}=0$ for the entire $\mathrm{C} 1-\mathrm{C} 2-\mathrm{C} 3-\mathrm{C} 4-\mathrm{C} 5$ chain indicates the presence of bonding that possesses the deformation characteristics of flexible closed-shell $B C P$ bonding with $\mathrm{Q}_{\text {rigidity }}=0.000$.

For a geometric dihedral angle $=0.0^{\mathrm{O}}$ there is no variation of the degree of the wrapping of the $\left\{\boldsymbol{q}, \boldsymbol{q}^{\prime}\right\}$ pathpackets, as measured by the precession $\mathrm{K}^{\prime}$ around the $\mathrm{C}-\mathrm{C} B C P$ bond-paths $\mathrm{r}$ for the [4]cumulene and S-1,5-dimethyl-[4]cumulene molecular graphs, see Figure 2(a), Figure 2(b). The absence of wrapping of the $\left\{\boldsymbol{q}, \boldsymbol{q}^{\prime}\right\}$ path-packets around the C-C $B C P$ bond-paths $\mathrm{r}$ along the C1-C2-C3-C4-C5 chain for $=0.0^{\mathbf{o}}$ is apparent from the identical values of $\mathrm{Q}_{\text {rigidity }}=1.000$, see Table 2 . This is not the case however, for the corresponding values of the $(-) \mathrm{S}(-),(+) \mathrm{S}(-)$ and $(+) \mathrm{S}(+)$ conformations of $\mathrm{S}-1,5$-diamino-[4]cumulene molecular graph at $=0.0^{\mathrm{o}}$ as demonstrated by the values $\mathrm{Q}_{\text {rigidity }}<1.000$, see Table 2and Figure 2(c-e) . For values of $=15.0^{\circ}$ and $75.0^{\circ}$ the bonding becomes increasingly polarized as demonstrated by the alternating high-low values of $\mathrm{Q}_{\text {rigidity }}$.

At $=90.0^{\circ}$ the $[4]$ cumulene, S-1,5-dimethyl-[4]cumulene and the $(-) \mathrm{S}(-),(+) \mathrm{S}(-)$ and $(+) \mathrm{S}(+)$ conformations of S-1,5-diamino-[4]cumulene possess the most polarized character bonding with the vast majority of values of $\mathrm{K}^{\prime}=1$ or $\mathrm{K}^{\prime}=0$, indicating a switch between strong and weak chemical character, this effect being most 
apparent for the [4]cumulene and S-1,5-dimethyl-[4]cumulene molecular graphs, see Figure 2 and Table 2 . To summarize, precession K' clearly identifies symmetry-breaking properties, from the asymmetrical form of $\mathrm{K}^{\prime}$ at $=15.0^{\mathrm{O}}$ for [4] cumulene and S-1,5-dimethyl-[4]cumulene and at $=0.0^{\mathrm{O}} 15.0^{\mathrm{O}}$ and $75.0^{\mathrm{O}}$ for the $(-) \mathrm{S}(-),(+) \mathrm{S}(-)$ and $(+) \mathrm{S}(+)$ conformations of S-1,5-diamino-[4]cumulene. The precession K' does notidentify symmetry-breaking for all values of or identify any helical characteristic previously identified by the helical frontier molecular orbital ${ }^{55}$. In the next section we will therefore use the stress tensor trajectory $\mathrm{T}_{\sigma}(s)_{\max }$ to investigate the presence of helical character as well as chirality.

Table 2. The values of $\mathrm{Q}_{\text {rigidity }}=\mathrm{Q} / \mathrm{Q}_{\text {maximum }}$ for [4]cumulene, S-1,5-dimethyl-[4]cumulene and the (-)S(), (+) $\mathrm{S}(-)$ and $(+) \mathrm{S}(+)$ conformations of $\mathrm{S}-1,5$-diamino-[4]cumulene and geometric angle , $\mathrm{Q}$ is the area enclosed by $\mathrm{K}$ ' for each $\mathrm{C}-\mathrm{C} B C P$ bond-path and $\mathrm{Q}_{\text {maximum }}$ corresponds to the area under $\mathrm{K}^{\prime}=1$ for each C-C $B C P$ bond-path.

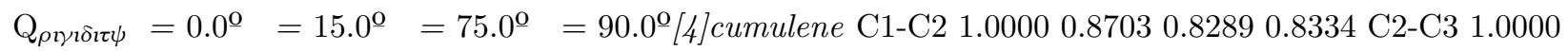
$0.49750 .07360 .0282 \mathrm{C} 3$-C4 1.00000 .91070 .96580 .9801 C4-C5 1.00000 .74130 .20800 .1646 S-1,5-dimethyl[4]cumulene C1-C2 $0.9997 \quad 0.8640 \quad 0.8475 \quad 0.8498$ C2-C3 $1.0000 \quad 0.38920 .0861 \quad 0.0640$ C3-C4 1.00000 .8762 0.93340 .9335 C4-C5 0.99980 .65800 .18230 .1522 (-)S(-) S-1,5-diamino-[4]cumulene C1-C2 0.60170 .7208

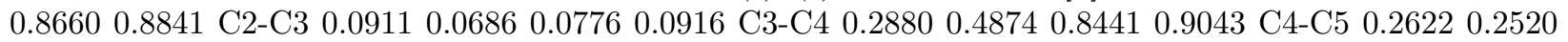
$0.14800 .1196(+) S(-)$ S-1,5-diamino-[4]cumulene C1-C2 0.62880 .74980 .88170 .8838 C2-C3 0.10770 .0776 0.08450 .0838 C3-C4 0.3013 0.5099 0.8859 0.9111 C4-C5 $0.26290 .24440 .14380 .1174(+) S(+)$ S-1,5-diamino[4]cumulene C1-C2 $0.61100 .74820 .8827 \quad 0.8053$ C2-C3 $0.1077 \quad 0.07480 .07940 .0909$ C3-C4 0.28190 .5061 0.88160 .9047

C4-C5 0.22290 .11010 .10960 .1220

\subsection{The chirality-helicity function $C_{\text {helicity }}$ \\ of [4]cumulene and variants}

The bond-twist $\mathrm{T}_{\sigma}$ quantifies the non-axialdisplacement of the $B C P$ and is defined as the difference (CCW minus $\mathrm{CW}$ ) of the bond-twist $\max$ component of the $\mathrm{T}_{\sigma}(s)_{\max }$. The bond-flexing $\mathrm{F}_{\sigma}$ provides a measure of the 'flexing-strain' and is defined as the difference (CCW minus $\mathrm{CW}$ ) of the bond-flexing max component of the stress tensor trajectory $\mathrm{T}_{\sigma}(s)_{\max }$. The chiral asymmetry (axiality) $\mathrm{A}_{\sigma}$ quantifies the degree of axial displacement $B C P$ along the bond-path and is defined as the difference (CCW minus $\mathrm{CW}$ ) of the bondaxiality $_{\max }$ component of the stress tensor trajectory $\mathrm{T}_{\sigma}(s)_{\max }$, see Table 3and Figure 3 . The axiality $\mathrm{A}_{\sigma}$ is the response to the bond torsion, i.e. the sliding of the $B C P$ along the bond-path ${ }^{51}$. The chirality-helicity function $\mathrm{C}_{\text {helicity }}=\mathrm{T}_{\sigma} \mathrm{A}_{\sigma}$ is defined as the simple arithmetic product of the non-axial $\left(\mathrm{T}_{\sigma}\right)$ and axial $\left(\mathrm{A}_{\sigma}\right)$ contributions of the $B C P$ displacement in response to the torsion $\vartheta$. For comparison purposes, we examined conventionally chiral (R)-1-chloro-1-phenylethane ${ }^{58}$ and found the chirality $\mathrm{C}_{\sigma}=-0.9859\left[\mathbf{P}_{\sigma}\right]$ for the chiral carbon atom, the axiality $\mathrm{A}_{\sigma}$ (previously referred to as the helicity $\mathrm{B}_{\sigma}$ ) $=-0.0110\left[\mathbf{P}_{\sigma}\right]$ and the $\mathrm{C}_{\text {helicity }}=$ 0.0108 .

Note the use of the term "chirality $\mathrm{C}_{\sigma}$ " which is reserved for use for a single chiral atom in a molecule which is not the case for the cumulenes where we are investigating the $\mathrm{C} 1-\mathrm{C} 2-\mathrm{C} 3-\mathrm{C} 4-\mathrm{C} 5$ chain of $\mathrm{BCP} \mathrm{s}$, however the method of calculation for the bond-twist $\mathrm{T}_{\sigma}$ is the same. For [4]cumulene the values of the bond-twist $\mathrm{T}_{\sigma}$ and bond-flexing $\mathrm{F}_{\sigma}$ are both insignificant for the $\mathrm{C} 1-\mathrm{C} 2-\mathrm{C} 3-\mathrm{C} 4-\mathrm{C} 5$ chain however, the axiality $\mathrm{A}_{\sigma}=$ 0.366 is very large compared with that of the previously calculated conventionally chiral (R)-1-chloro-1phenylethane. Examination of the $\mathrm{T}_{\sigma}(s)$ along the bond-axiality axis, used to calculate the axiality $\mathrm{A}_{\sigma}$, demonstrates the dominance of the axiality $\mathrm{A}_{\sigma}$ and the lack of bond-twist $\mathrm{T}_{\sigma}$, see Figure $\mathbf{3}$. The S-1,5dimethyl-[4]cumulene possesses rather low values of $\mathrm{T}_{\sigma}$ and $\mathrm{C}_{\text {helicity }}$ for the $\mathrm{C} 1-\mathrm{C} 2-\mathrm{C} 3-\mathrm{C} 4-\mathrm{C} 5$ chain, however the values of $\mathrm{T}_{\sigma}$ and therefore $\mathrm{C}_{\text {helicity }}$ are an order of magnitude higher for the $\mathrm{C} 1-\mathrm{C} 2$ BCP and the C2-C3 $B C P$. We note that the $(-) \mathrm{S}(-),(+) \mathrm{S}(-)$ and $(+) \mathrm{S}(+)$ conformations of $\mathrm{S}-1,5$-diamino-[4]cumulene all possess greater $\mathrm{C}_{\text {helicity }}$ values for the terminal C1-C2 BCP and C4-C5 BCP than the central C2-C3 BCP and C3-C4 $B C P$. The maximum stress tensor projections $\left\{\right.$ bond-twist $\max$, bond-flexing $\max _{\text {ax }}$, bond-axiality $\left.\max \right\}$ and the bond-flexing $\mathrm{F}_{\sigma}$ of the cumulene variants are presented in the Supplementary Materials S5 . 
Table 3. The values of the bond-twist $T_{\sigma}$, bond-flexing $F_{\sigma}$ and the axiality $A_{\sigma}$ and of the chirality-helicity function $\mathrm{C}_{\text {helicity }}=$ (bond-twist $\mathrm{T}_{\sigma}$ ) (axiality $\mathrm{A}_{\sigma}$ ) for the $\mathrm{C}-\mathrm{C} B C P s$ of the unsubstituted and substituted cumulenes, seeScheme 1 and the Table S5(a) of theSupplementary Materials S5 .

$\left\{\mathrm{T}_{\sigma}, \quad \mathrm{F}_{\sigma}, \quad \mathrm{A}_{\sigma}\right\} \quad \mathrm{C}_{\text {helicity }}\left[\mathrm{T}_{\sigma}, \mathrm{A}_{\sigma}\right][4]$ cumulene $\mathrm{C} 1-\mathrm{C} 2 \quad B C P \quad\left\{0.00000\left[\boldsymbol{\Sigma}_{\sigma}\right], \quad 0.00001\left[\boldsymbol{\Sigma}_{\sigma}\right], \quad 0.36621\left[\boldsymbol{\Sigma}_{\sigma}\right]\right\}$ $0.00000\left[\boldsymbol{\Sigma}_{\boldsymbol{\sigma}}, \boldsymbol{\Sigma}_{\boldsymbol{\sigma}}\right] \mathrm{C} 2-\mathrm{C} 3 \quad B C P \quad\left\{0.00001\left[\boldsymbol{\Sigma}_{\boldsymbol{\sigma}}\right], \quad-0.00002\left[\mathbf{P}_{\boldsymbol{\sigma}}\right], \quad 0.58335\left[\boldsymbol{\Sigma}_{\boldsymbol{\sigma}}\right]\right\} \quad 0.00001\left[\boldsymbol{\Sigma}_{\boldsymbol{\sigma}}, \boldsymbol{\Sigma}_{\boldsymbol{\sigma}}\right] \mathrm{C} 3-\mathrm{C} 4$ $B C P \quad\left\{-0.00002\left[\mathbf{P}_{\boldsymbol{\sigma}}\right], \quad 0.00001\left[\boldsymbol{\Sigma}_{\boldsymbol{\sigma}}\right], \quad 0.58336\left[\boldsymbol{\Sigma}_{\boldsymbol{\sigma}}\right]\right\} \quad-0.00001\left[\mathbf{P}_{\boldsymbol{\sigma}}, \boldsymbol{\Sigma}_{\boldsymbol{\sigma}}\right] \mathrm{C} 4-\mathrm{C} 5 \quad B C P \quad\left\{-0.00006\left[\mathbf{P}_{\boldsymbol{\sigma}}\right]\right.$, $\left.0.00000\left[\boldsymbol{\Sigma}_{\boldsymbol{\sigma}}\right], \quad 0.36633\left[\boldsymbol{\Sigma}_{\boldsymbol{\sigma}}\right]\right\} \quad-0.00002\left[\mathbf{P}_{\boldsymbol{\sigma}}, \boldsymbol{\Sigma}_{\boldsymbol{\sigma}}\right]$ S-1,5-dimethyl-[4]cumuleneC1-C2 BCP $\quad\left\{-0.02677\left[\mathbf{P}_{\boldsymbol{\sigma}}\right]\right.$, $\left.-0.04781\left[\mathbf{P}_{\boldsymbol{\sigma}}\right], \quad 0.18036\left[\boldsymbol{\Sigma}_{\boldsymbol{\sigma}}\right]\right\} \quad-0.00483\left[\mathbf{P}_{\boldsymbol{\sigma}}, \boldsymbol{\Sigma}_{\boldsymbol{\sigma}}\right] \mathrm{C} 2-\mathrm{C} 3 \quad B C P \quad\left\{-0.00858\left[\mathbf{P}_{\boldsymbol{\sigma}}\right],-0.01873\left[\mathbf{P}_{\boldsymbol{\sigma}}\right], \quad 0.33567\left[\boldsymbol{\Sigma}_{\boldsymbol{\sigma}}\right]\right\}$ $-0.00288\left[\mathbf{P}_{\boldsymbol{\sigma}}, \boldsymbol{\Sigma}_{\boldsymbol{\sigma}}\right] \mathrm{C} 3-\mathrm{C} 4 \quad B C P \quad\left\{0.00052\left[\boldsymbol{\Sigma}_{\boldsymbol{\sigma}}\right],-0.00093\left[\mathbf{P}_{\boldsymbol{\sigma}}\right], \quad 0.34030\left[\boldsymbol{\Sigma}_{\boldsymbol{\sigma}}\right]\right\} \quad 0.00018\left[\boldsymbol{\Sigma}_{\boldsymbol{\sigma}}, \boldsymbol{\Sigma}_{\boldsymbol{\sigma}}\right] \mathrm{C} 4-\mathrm{C} 5 \quad B C P$ $\left\{-0.00282\left[\mathbf{P}_{\sigma}\right], \quad 0.00050\left[\boldsymbol{\Sigma}_{\boldsymbol{\sigma}}\right], \quad 0.17749\left[\boldsymbol{\Sigma}_{\boldsymbol{\sigma}}\right]\right\} \quad-0.00050\left[\mathbf{P}_{\boldsymbol{\sigma}}, \boldsymbol{\Sigma}_{\boldsymbol{\sigma}}\right](-) S(-) \quad$ S-1,5-diamino-[4]cumuleneC1$\mathrm{C} 2 \quad B C P \quad\left\{0.24690\left[\boldsymbol{\Sigma}_{\boldsymbol{\sigma}}\right], \quad-0.21197\left[\mathbf{P}_{\sigma}\right], \quad 1.28073\left[\boldsymbol{\Sigma}_{\boldsymbol{\sigma}}\right]\right\} \quad 0.31621\left[\boldsymbol{\Sigma}_{\boldsymbol{\sigma}}, \boldsymbol{\Sigma}_{\boldsymbol{\sigma}}\right] \mathrm{C} 2-\mathrm{C} 3 \quad B C P \quad\left\{-0.14710\left[\mathbf{P}_{\sigma}\right]\right.$, $\left.0.04266\left[\boldsymbol{\Sigma}_{\boldsymbol{\sigma}}\right],-0.45718\left[\mathbf{P}_{\sigma}\right]\right\} \quad 0.06725\left[\mathbf{P}_{\boldsymbol{\sigma}}, \mathbf{P}_{\boldsymbol{\sigma}}\right] \mathrm{C} 3-\mathrm{C} 4 \quad B C P \quad\left\{0.14262\left[\boldsymbol{\Sigma}_{\boldsymbol{\sigma}}\right],-0.04699\left[\mathbf{P}_{\sigma}\right],-0.45292\left[\mathbf{P}_{\sigma}\right]\right\}$ $-0.06460\left[\boldsymbol{\Sigma}_{\boldsymbol{\sigma}}, \mathbf{P}_{\sigma}\right] \mathrm{C} 4-\mathrm{C} 5 \quad B C P \quad\left\{0.31485\left[\boldsymbol{\Sigma}_{\boldsymbol{\sigma}}\right], \quad-0.19825\left[\mathbf{P}_{\boldsymbol{\sigma}}\right], \quad 1.25469\left[\boldsymbol{\Sigma}_{\boldsymbol{\sigma}}\right]\right\} \quad 0.39561\left[\boldsymbol{\Sigma}_{\boldsymbol{\sigma}}, \boldsymbol{\Sigma}_{\boldsymbol{\sigma}}\right](+) S(-)$ S-1,5-diamino-[4]cumuleneC1-C2 BCP $\left\{0.17738\left[\boldsymbol{\Sigma}_{\boldsymbol{\sigma}}\right],-0.66454\left[\mathbf{P}_{\boldsymbol{\sigma}}\right],-1.80066\left[\mathbf{P}_{\sigma}\right]\right\}-0.31940\left[\boldsymbol{\Sigma}_{\boldsymbol{\sigma}}, \mathbf{P}_{\sigma}\right] \mathrm{C} 2-$ C3 BCP $\left\{-0.01920\left[\mathbf{P}_{\sigma}\right], \quad-0.02399\left[\mathbf{P}_{\sigma}\right], \quad-1.03702\left[\mathbf{P}_{\sigma}\right]\right\} \quad 0.01991\left[\mathbf{P}_{\boldsymbol{\sigma}}, \mathbf{P}_{\sigma}\right] C 3-C 4 \quad B C P \quad\left\{-0.08852\left[\mathbf{P}_{\boldsymbol{\sigma}}\right]\right.$, $\left.-0.06836\left[\mathbf{P}_{\sigma}\right], \quad-1.01975\left[\mathbf{P}_{\sigma}\right]\right\} \quad 0.09027\left[\mathbf{P}_{\boldsymbol{\sigma}}, \mathbf{P}_{\sigma}\right] \mathrm{C} 4-\mathrm{C} 5 \quad B C P \quad\left\{0.08485\left[\boldsymbol{\Sigma}_{\boldsymbol{\sigma}}\right], \quad-0.17413\left[\mathbf{P}_{\boldsymbol{\sigma}}\right]\right.$,

$\left.1.86474\left[\mathbf{P}_{\sigma}\right]\right\} \quad-0.15822\left[\boldsymbol{\Sigma}_{\boldsymbol{\sigma}}, \mathbf{P}_{\sigma}\right](+) S(+) \quad$ S-1,5-diamino-[4]cumulene $\quad$ C1-C2 $B C P \quad\left\{0.86363\left[\boldsymbol{\Sigma}_{\boldsymbol{\sigma}}\right], \quad-\right.$ $\left.0.21694\left[\mathbf{P}_{\sigma}\right], \quad 6.74465\left[\boldsymbol{\Sigma}_{\boldsymbol{\sigma}}\right]\right\} \quad 5.82488\left[\boldsymbol{\Sigma}_{\boldsymbol{\sigma}}, \boldsymbol{\Sigma}_{\boldsymbol{\sigma}}\right] \mathrm{C} 2-\mathrm{C} 3 \quad B C P \quad\left\{-0.16848\left[\mathbf{P}_{\sigma}\right],-0.23924\left[\mathbf{P}_{\sigma}\right],-7.77013\left[\mathbf{P}_{\sigma}\right]\right\}$ $1.30911\left[\mathbf{P}_{\boldsymbol{\sigma}}, \mathbf{P}_{\boldsymbol{\sigma}}\right] \mathrm{C} 3-\mathrm{C} 4 \quad B C P \quad\left\{-0.18147\left[\mathbf{P}_{\boldsymbol{\sigma}}\right],-0.34965\left[\mathbf{P}_{\boldsymbol{\sigma}}\right],-7.77102\left[\mathbf{P}_{\boldsymbol{\sigma}}\right]-1.41021\left[\mathbf{P}_{\boldsymbol{\sigma}}, \mathbf{P}_{\boldsymbol{\sigma}}\right] \mathrm{C} 4-\mathrm{C} 5 \quad B C P\right.$ $\left\{0.98399\left[\boldsymbol{\Sigma}_{\sigma}\right], 0.98398\left[\boldsymbol{\Sigma}_{\sigma}\right], 6.70333\left[\boldsymbol{\Sigma}_{\sigma}\right]\right\} 6.59601\left[\boldsymbol{\Sigma}_{\boldsymbol{\sigma}}, \boldsymbol{\Sigma}_{\boldsymbol{\sigma}}\right]$

The linear stress tensor trajectories $\mathrm{T}_{\sigma}(s)$ plot for [4]cumulene reflects the presence pure axial motion, i.e. only values of axiality $\left|\mathrm{A}_{\sigma}\right|>0$ are present and are created by $B C P$ sliding along the bond-path of the $B C P$, is a consequence of the value of the bond-twist $\mathrm{T}_{\sigma}=0.0$, see the Supplementary Materials S5and Table 3 . Significant values of the $\mathrm{C}_{\text {helicity }}$ are evident from twisting of stress tensor trajectories $\mathrm{T}_{\sigma}(s)$ plots for S-1,5- dimethyl-[4]cumulene, see Figure 3(a). The very large values of the chirality-helicity function $\mathrm{C}_{\text {helicity }}$ are evident from the large open spiral form of the stress tensor trajectories $\mathrm{T}_{\sigma}(s)$ plots for the $(-) \mathrm{S}(-),(+) \mathrm{S}(-)$ and $(+) \mathrm{S}(+)$ conformations of S-1,5-diamino-[4]cumulene, see Figure 4(a-c) respectively. 


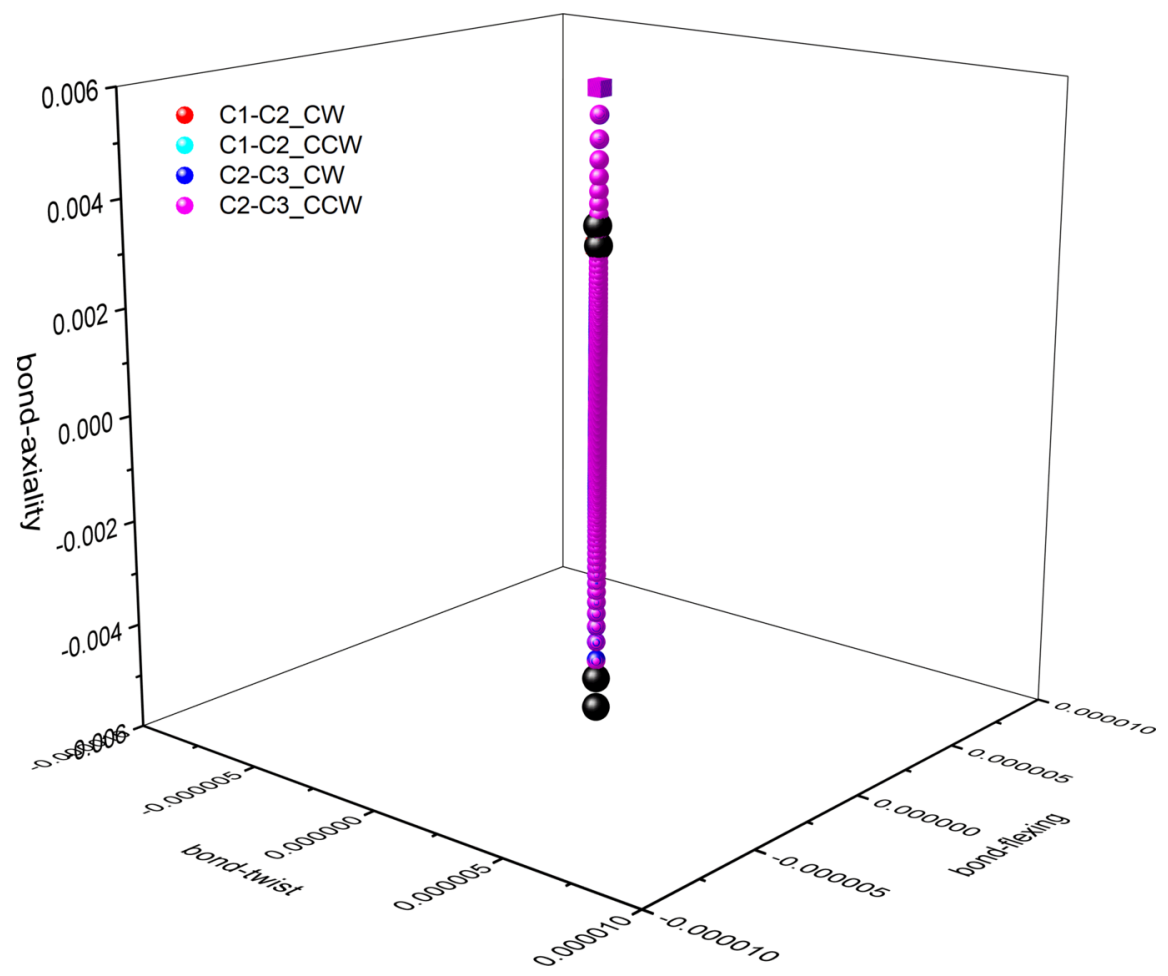

(a)

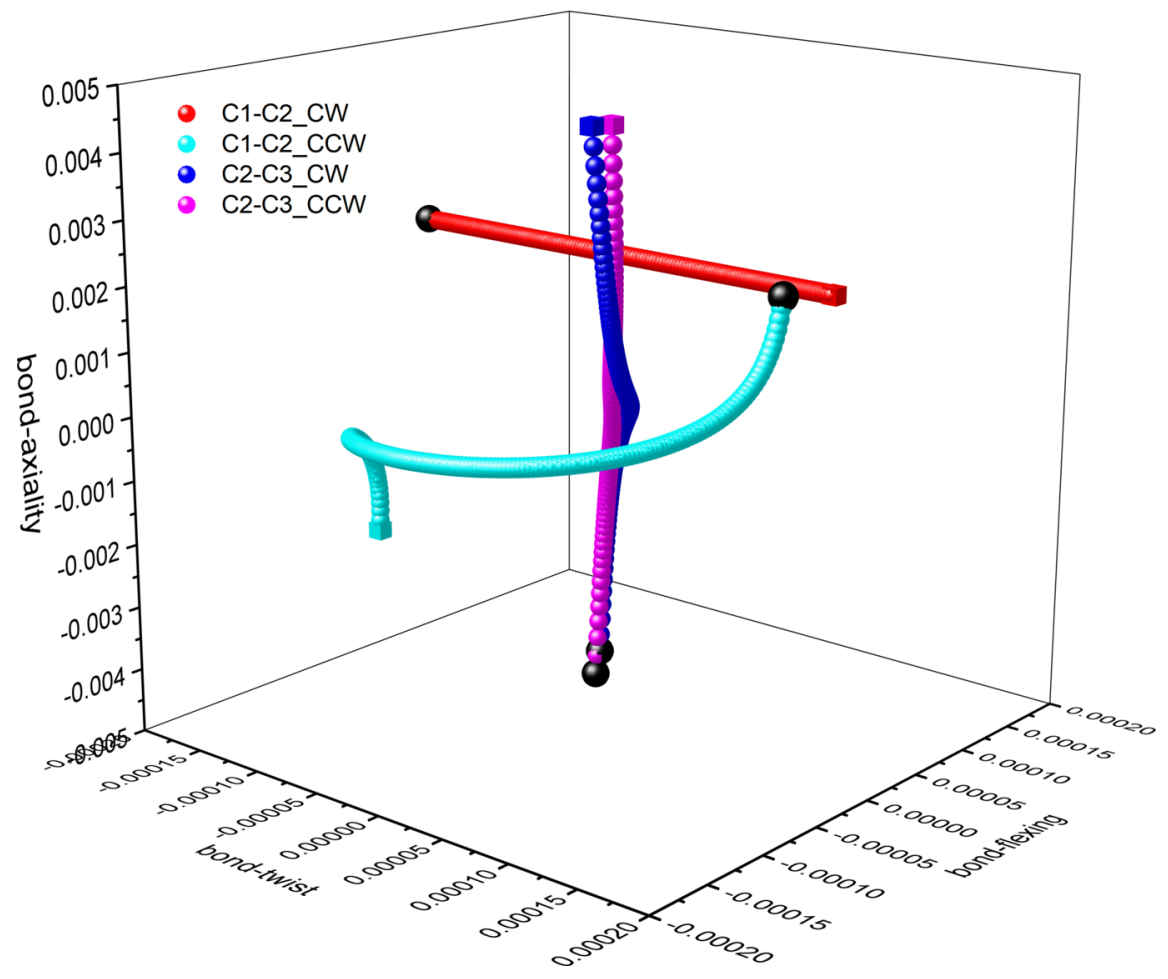


(b)

Figure 3. The stress tensor trajectories $\mathrm{T}_{\sigma}(s)$ of the $\mathrm{C} 1-\mathrm{C} 2 B C P, \mathrm{C} 2-\mathrm{C} 3 B C P, \mathrm{C} 3-\mathrm{C} 4 B C P$ and C4-C5 BCP [4]cumulene and S-1,5- dimethyl-[4]cumulene are presented in each of sub-figures(a) and (b) respectively. The end of each $\mathrm{T}_{\sigma}(s)$ is denoted by a cube marker.

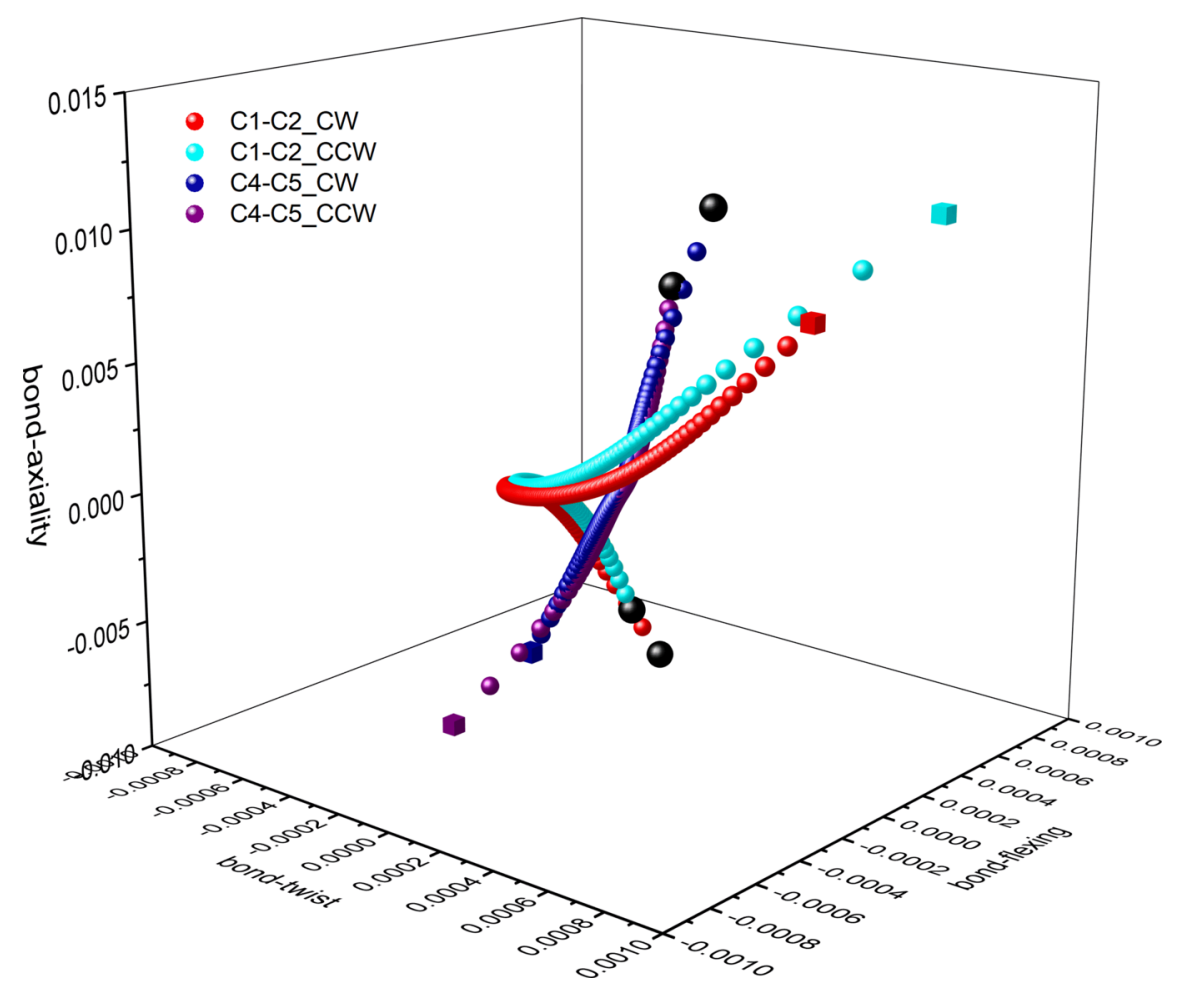

(a) 

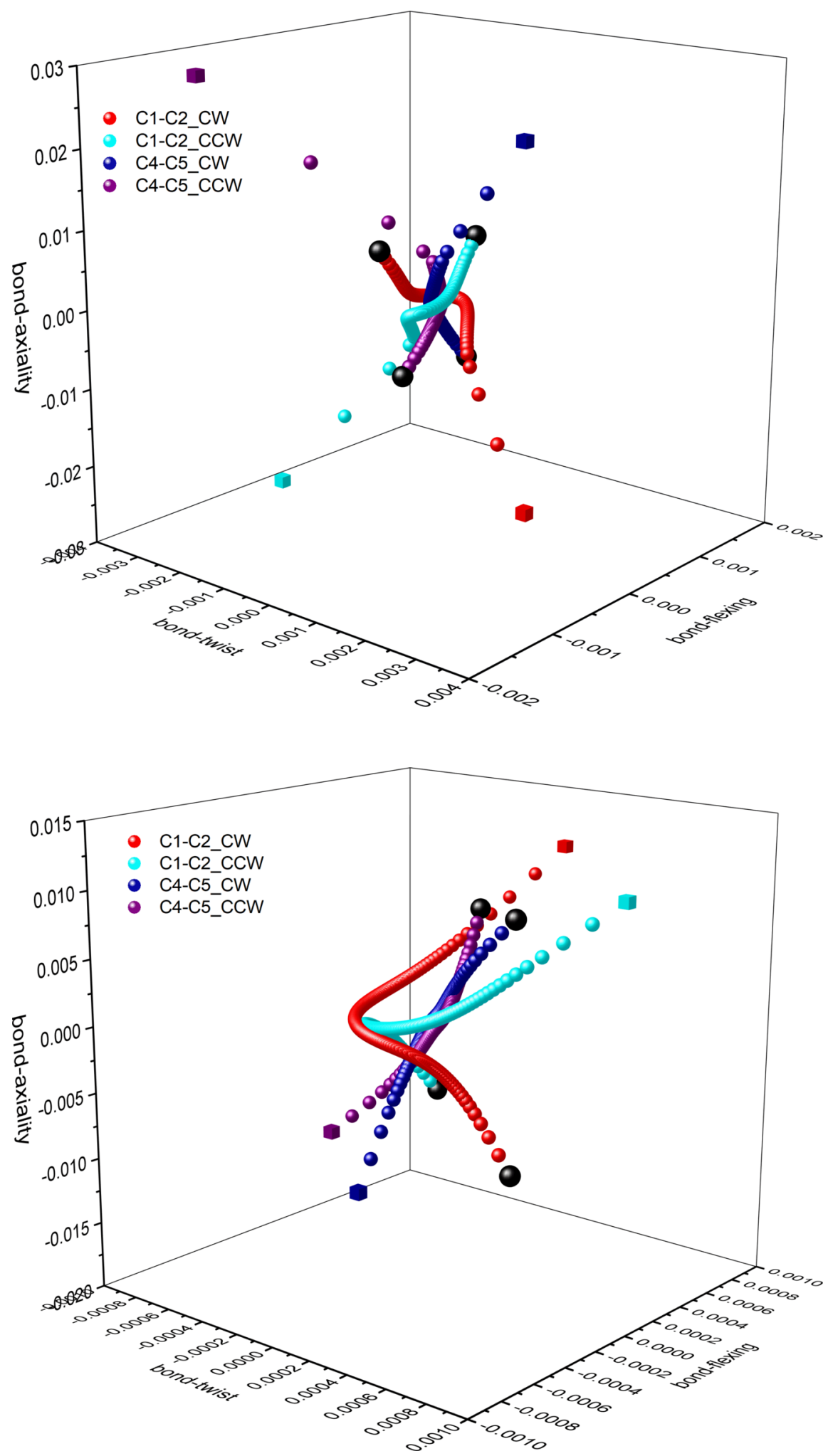

(b) 
(c)

Figure 4. The stress tensor trajectories $\mathrm{T}_{\sigma}(s)$ of the $\mathrm{C} 1-\mathrm{C} 2 B C P, \mathrm{C} 4-\mathrm{C} 5 \mathrm{BCP}$ (left panel) and $\mathrm{C} 2-\mathrm{C} 3 \mathrm{BCP}$ , C3-C4 $B C P$ (right panel) of the $(-) \mathrm{S}(-),(+) \mathrm{S}(-)$ and $(+) \mathrm{S}(+)$ conformations of S-1,5-diamino-[4]cumulene are presented in each of sub-figures(a-c) respectively. The end of each $\mathrm{T}_{\sigma}(s)$ is denoted by a cube marker.

\section{Conclusions}

In this investigation we visualized the bond-path framework set $\mathrm{B}\left\{\mathrm{q}, \mathrm{q}^{\prime}, \mathrm{r}\right\}$ for geometric dihedral angles $=$ $0.0^{\circ}, 15.0^{\circ}, 75.0^{\circ}$ and $90.0^{\circ}$ of [4] cumulene, S-1,5-dimethyl-[4]cumulene and the $(-) \mathrm{S}(-),(+) \mathrm{S}(-)$ and $(+) \mathrm{S}(+)$ conformations of S-1,5-diamino-[4]cumulene. There we saw a variation of the precession $\mathrm{K}$ for the cumulene variants and dihedral angle, where $\mathrm{K}$ quantifies the wrapping of the $\left\{\mathrm{q}, \mathrm{q}^{\prime}\right\}$ path-packets around the bondpath $\mathrm{r}$ and the $\left\{\mathrm{q}, \mathrm{q}^{\prime}\right\}$ path-packets are constructed from the directions of most preferred electronic motion. The directional character of the chemical bonding is then extracted as the $\mathrm{Q}_{\text {rigidity }}$ that is used to explain the lack of twisting of the $\left\{q, q^{\prime}\right\}$ path-packets of [4]cumulene and S-1,5-dimethyl-[4]cumulene around the bond-path $\mathrm{r}$ that occurs for the maximum possible value of $\mathrm{Q}_{\text {rigidity }}(=1.0)$ for $=0.0^{\mathrm{O}}$. In addition, for all the cumulene variants the $\mathrm{Q}_{\text {rigidity }}$ quantifies a strong trend towards polarization of the bonding between the possession of deformation characteristics of weak and strong chemical bonds as the geometric dihedral angle is increased to $=90.0^{\circ}$.

We have demonstrated that the presence of the chirality-helicity equivalence by observing and quantifying helical shaped stress tensor trajectories $\mathrm{T}_{\sigma}(s)$ for the S-1,5-dimethyl-[4]cumulene, $(-) \mathrm{S}(-),(+) \mathrm{S}(-)$ and $(+) \mathrm{S}(+)$ conformations of S-1,5-diamino-[4]cumulene. We used the stress tensor trajectories $\mathrm{T}_{\sigma}(s)$ to determine the bond-twist $\mathrm{T}_{\sigma}$, the axiality $\mathrm{A}_{\sigma}$ and the chirality-helicity function $\mathrm{C}_{\text {helicity }}$. Very large values of the chirality-helicity function $\mathrm{C}_{\text {helicity }}$ are evident from the helix form of the stress tensor trajectories $\mathrm{T}_{\sigma}(s$ ), this finding is consistent with the helical orbitals discovered in earlier investigations into lactic acid and alanine $e^{20}$.

The largest bond-twist $\mathrm{T}_{\sigma}$ values occur for the end-point C1-C2 BCP and C4-C5 BCP of the (-)S(-), (+)S(-) and $(+) \mathrm{S}(+)$ conformations of S-1,5-diamino-[4]cumulene and corresponds to the presence of $\boldsymbol{\Sigma}_{\boldsymbol{\sigma}}$ chirality and $\boldsymbol{\Sigma}_{\boldsymbol{\sigma}}$ axiality. The chirality-helicity function $\mathrm{C}_{\text {helicity }}$ of S-1,5-dimethyl-[4]cumulene comprises a mix of $\boldsymbol{\Sigma}_{\boldsymbol{\sigma}}$ chirality and $\mathbf{P}_{\boldsymbol{\sigma}}$ axiality and the low values of bond-twist $\mathrm{T}_{\sigma}$ but significant axiality $\mathrm{A}_{\sigma}$ results in the morphology of the stress tensor trajectories $\mathrm{T}_{\sigma}(s)$ being weakly helical.

[4] Cumulene is the first molecular graph we have discovered that possesses an absence of bond-twist $\mathrm{T}_{\sigma}$ and a very large value of the axiality $\mathrm{A}_{\sigma}$ and consequently a negligible value of the chirality-helicity function $\mathrm{C}_{\text {helicity }}$. We do not therefore expect the presence of chiral and helical properties in cumulene[4] to be discoverable by experiments such as those undertaken by Beaulieuet al. on neutral molecules ${ }^{19}$ due to the insignificant degree of the chirality-helicity function $\mathrm{C}_{\text {helicity }}$. We do however expect, on the basis of the very large values of the chirality-helicity function $\mathrm{C}_{\text {helicity }}$, that the chiral and helical properties of the $(-) \mathrm{S}(-),(+) \mathrm{S}(-)$ and $(+) \mathrm{S}(+)$ conformations of S-1,5-diamino-[4]cumulene would be discoverable by optical experiments.

\section{Acknowledgements}

The National Natural Science Foundation of China is acknowledged, project approval number: 21673071. The One Hundred Talents Foundation of Hunan Province are gratefully acknowledged for the support of S.J. and S.R.K. We thank Marc H. Garner for initial energy minimized and torsioned cumulene structures.

\section{References}

1. K. C. Harper and M. S. Sigman, Science, 2011, 333, 1875-1878.

2. M. H. Garner and C. Corminboeuf, Chem. Commun., 2021, 57, 6408-6411.

3. R. S. Cahn, C. Ingold and V. Prelog, Angew. Chem. Int. Ed. Engl. , 1966 , 5, 385-415.

4. V. Prelog and G. Helmchen, Angew. Chem. Int. Ed. Engl. ,1982, 21, 567-583. 
5. M. H. Garner, R. Hoffmann, S. Rettrup and G. C. Solomon, ACS Cent. Sci. , 2018 , 4, 688-700.

6. R. J. Buenker, J. Chem. Phys., 1968, 48, 1368-1379.

7. H. Dickerson, S. Ferber and F. S. Richardson, Theoret. Chim. Acta , 1976 , 42, 333-344.

8. E. Deretey, M. Shapiro and P. Brumer, J. Phys. Chem. A ,2001 , 105, 9509-9517.

9. X. Gu, R. I. Kaiser and A. M. Mebel, ChemPhysChem ,2008 , 9, 350-369.

10. L. Ravagnan, N. Manini, E. Cinquanta, G. Onida, D. Sangalli, C. Motta, M. Devetta, A. Bordoni, P. Piseri and P. Milani, Phys. Rev. Lett. , 2009 , 102, 245502.

11. A. Imamura and Y. Aoki, Chemical Physics Letters ,2013 , 590, 136-140.

12. C. H. Hendon, D. Tiana, A. T. Murray, D. R. Carbery and A. Walsh, Chem. Sci. , 2013 , 4, 4278-4284.

13. A. Fresnel, Mémoires de l'Académie des sciences de l'Institut de France , 1821 , 7, 45-176.

14. J. H. Brewster, in Stereochemistry I , Springer, Berlin, Heidelberg, 1974 , pp. 29-71.

15. L. Rosenfeld, Z. Physik, 1929, 52, 161-174.

16. D. J. Caldwell, H. Eyring and T. Y. Chang, Physics Today ,1972 , 25, 53.

17. I. Tinoco and R. W. Woody, The Journal of Chemical Physics ,1964 , 40, 160-165.

18. D. Zhigang Wang, Mendeleev Communications , 2004 , 14, 244-247.

19. S. Beaulieu, A. Comby, D. Descamps, B. Fabre, G. A. Garcia, R. Géneaux, A. G. Harvey, F. Légaré, Z. Mašín, L. Nahon, A. F. Ordonez, S. Petit, B. Pons, Y. Mairesse, O. Smirnova and V. Blanchet, Nature Phys , $2018,14,484-489$.

20. T. Xu, J. H. Li, R. Momen, W. J. Huang, S. R. Kirk, Y. Shigeta and S. Jenkins, J. Am. Chem. Soc. , $2019,141,5497-5503$.

21. K. Banerjee-Ghosh, O. Ben Dor, F. Tassinari, E. Capua, S. Yochelis, A. Capua, S.-H. Yang, S. S. P. Parkin, S. Sarkar, L. Kronik, L. T. Baczewski, R. Naaman and Y. Paltiel, Science, 2018 , 360, 1331-1334.

22. R. F. W. Bader, Atoms in Molecule: A Quantum Theory, Oxford University Press, Oxford, UK, 1990 , vol. 22 .

23. C. T. Haakansson, T. R. Corkish, P. D. Watson, H. T. Robinson, T. Tsui, A. J. McKinley and D. A. Wild, ChemPhysChem , 2021 , 22, 806-806.

24. R. F. W. Bader, The Journal of Chemical Physics ,1980 , 73, 2871-2883.

25. T. Xu, S. R. Kirk and S. Jenkins, Chemical Physics Letters ,2020 , 738, 136907.

26. R. F. W. Bader, J. Phys. Chem. A, 2009 , 113, 10391-10396.

27. P. W. Ayers and S. Jenkins, The Journal of Chemical Physics ,2009 , 130, 154104.

28. S. Jenkins, S. R. Kirk, A. S. Côté, D. K. Ross and I. Morrison, Can. J. Phys., 2003 , 81, 225-231.

29. R. G. A. Bone and R. F. W. Bader, J. Phys. Chem. ,1996 , 100, 10892-10911.

30. S. Jenkins and M. I. Heggie, Journal of Physics: Condensed Matter , 2000 , 12, 10325-10333.

31. A. Azizi, R. Momen, H. Früchtl, T. van Mourik, S. R. Kirk and S. Jenkins, J Comput Chem, 2020 , 41, 913-921.

32. X. Bin, T. Xu, S. R. Kirk and S. Jenkins, Chemical Physics Letters , 2019 , 730, 506-512. 
33. T. Xu, R. Momen, A. Azizi, T. van Mourik, H. Früchtl, S. R. Kirk and S. Jenkins, Journal of Computational Chemistry, 2019, 40, 1881-1891.

34. T. Tian, T. Xu, S. R. Kirk, M. Filatov and S. Jenkins, Chemical Physics Letters, 2019 , 717, 91-98.

35. T. Tian, T. Xu, S. R. Kirk, M. Filatov and S. Jenkins, International Journal of Quantum Chemistry, 2019,119, e25862.

36. X. Bin, A. Azizi, T. Xu, S. R. Kirk, M. Filatov and S. Jenkins, International Journal of Quantum Chemistry, 2019, 119, e25957.

37. X. Bin, R. Momen, T. Xu, S. R. Kirk, M. Filatov and S. Jenkins, International Journal of Quantum Chemistry, 2019, 119, e25903.

38. T. Tian, T. Xu, T. van Mourik, H. Früchtl, S. R. Kirk and S. Jenkins, Chemical Physics Letters, 2019 , 722, 110-118.

39. T. Malcomson, A. Azizi, R. Momen, T. Xu, S. R. Kirk, M. J. Paterson and S. Jenkins, J. Phys. Chem. A, 2019, 123, 8254-8264.

40. S. Li, T. Xu, T. van Mourik, H. Früchtl, S. R. Kirk and S. Jenkins,Molecules, 2019 , 24, 2875.

41. A. Azizi, R. Momen, S. R. Kirk and S. Jenkins, Phys. Chem. Chem. Phys., 2020 , 22, 864-877.

42. Y. Yang, T. Xu, S. R. Kirk and S. Jenkins, International Journal of Quantum Chemistry, 2021, 121, $\mathrm{e} 26584$.

43. X. Nie, Y. Yang, T. Xu, S. R. Kirk and S. Jenkins, International Journal of Quantum Chemistry, 2021 , 121, e26527.

44. B. Mahara, A. Azizi, Y. Yang, M. Filatov, S. R. Kirk and S. Jenkins, Chemical Physics Letters , 2021 , 766,138339 .

45. T. A. Keith, AIMAll, Revision 19.10.12 , TK Gristmill Software, Overland Park KS, USA, 2019.

46. P. Yang, T. Xu, R. Momen, A. Azizi, S. R. Kirk and S. Jenkins, Int J Quantum Chem , 2018, 118, e25565.

47. T. Xu, L. Wang, Y. Ping, T. van Mourik, H. Früchtl, S. R. Kirk and S. Jenkins, Int. J. Quantum Chem. , 2018,118 , e25676.

48. T. Xu, J. Farrell, R. Momen, A. Azizi, S. R. Kirk, S. Jenkins and D. J. Wales, Chem. Phys. Lett., 2017 , 667, 25-31.

49. T. Xu, R. Momen, A. Azizi, T. van Mourik, H. Früchtl, S. R. Kirk and S. Jenkins, J. Comput. Chem. , $2019,40,1881-1891$.

50. T. Tian, T. Xu, T. van Mourik, H. Früchtl, S. R. Kirk and S. Jenkins, Chem. Phys. Lett., 2019 , 722, $110-118$.

51. T. Tian, T. Xu, S. R. Kirk, I. T. Rongde, Y. B. Tan, S. Manzhos, Y. Shigeta and S. Jenkins, Phys. Chem. Chem. Phys., 2020, 22, 2509-2520.

52. A. D. Becke and E. R. Johnson, The Journal of Chemical Physics , 2006 , 124, 221101.

53. S. Grimme, S. Ehrlich and L. Goerigk, Journal of Computational Chemistry , 2011, 32, 1456-1465.

54. Michael J. Frisch, G. W. Trucks, H. Bernhard Schlegel, Gustavo E. Scuseria, Michael A. Robb, James R. Cheeseman, Giovanni Scalmani, Vincenzo Barone, Benedetta Mennucci, G. A. Petersson, H. Nakatsuji, M. Caricato, Xiaosong Li, H. P. Hratchian, Artur F. Izmaylov, Julien Bloino, G. Zheng, J. L. Sonnenberg, M. Hada, M. Ehara, K. Toyota, R. Fukuda, J. Hasegawa, M. Ishida, T. Nakajima, Y. Honda, O. Kitao, H. Nakai, T. Vreven, J. A. Montgomery Jr., J. E. Peralta, François Ogliaro, Michael J. Bearpark, Jochen Heyd, E. N. 
Brothers, K. N. Kudin, V. N. Staroverov, Rika Kobayashi, J. Normand, Krishnan Raghavachari, Alistair P. Rendell, J. C. Burant, S. S. Iyengar, Jacopo Tomasi, M. Cossi, N. Rega, N. J. Millam, M. Klene, J. E. Knox, J. B. Cross, V. Bakken, C. Adamo, J. Jaramillo, R. Gomperts, R. E. Stratmann, O. Yazyev, A. J. Austin, R. Cammi, C. Pomelli, J. W. Ochterski, R. L. Martin, K. Morokuma, V. G. Zakrzewski, G. A. Voth, P. Salvador, J. J. Dannenberg, S. Dapprich, A. D. Daniels, Ö. Farkas, J. B. Foresman, J. V. Ortiz, J. Cioslowski and D. J. Fox, Gaussian 09, Revision E.01 , Gaussian, Inc., 340 Quinnipiac St Bldg 40 Wallingford, CT 06492 USA,2009 .

55. M. H. Garner, A. Jensen, L. O. H. Hyllested and G. C. Solomon, Chem. Sci. , 2019 , 10, 4598-4608.

56. S. R. Kirk and S. Jenkins, QuantVec, BEACON Research Group, College of Chemistry and Chemical Engineering, Hunan Normal University, Changsha, Hunan, P.R. China, 2021.

57. P. Ramachandran and G. Varoquaux, Computing in Science Engineering, 2011, 13, 40-51.

58. T. Xu, X. Nie, S. Li, Y. Yang, H. Früchtl, T. van Mourik, S. R. Kirk, M. J. Paterson, Y. Shigeta and S. Jenkins, ChemPhysChem ,2021 , 22, 1989-1995. 